\title{
LORENTZIAN AND EUCLIDEAN QUANTUM GRAVITY - ANALYTICAL AND NUMERICAL RESULTS
}

\author{
Lectures presented at the 1999 NATO-ASI on "Quantum Geometry" in \\ Akureyri, Iceland. \\ Preprint: NBI-HE-00-02, AEI-2000-0
}

\author{
J. AMBJØRN \\ The Niels Bohr Institute, \\ Blegdamsvej 17, DK-2100 Copenhagen Ø, Denmark \\ email: ambjorn@nbi.dk \\ J. JURKIEWICZ \\ Institute of Physics, Jagellonian University, \\ ul. Reymonta 4, PL-30 059, Kraków 16, Poland \\ email: jurkiewi@thrisc.if.uj.edu.pl \\ AND \\ R. LOLL \\ Albert Einstein Institute \\ (Max-Planck-Institute for Gravitational Physics), \\ Am Mühlenberg 1, D-14476 Golm, Germany \\ email: loll@aei-potsdam.mpg.de
}

\begin{abstract}
We review some recent attempts to extract information about the nature of quantum gravity, with and without matter, by quantum field theoretical methods. More specifically, we work within a covariant lattice approach where the individual space-time geometries are constructed from fundamental simplicial building blocks, and the path integral over geometries is approximated by summing over a class of piece-wise linear geometries. This method of "dynamical triangulations" is very powerful in $2 \mathrm{~d}$, where the regularized theory can be solved explicitly, and gives us more insights into the quantum nature of $2 \mathrm{~d}$ space-time than continuum methods are presently able to provide. It also allows us to establish an explicit relation between the Lorentzian- and Euclidean-signature quantum theories. Analogous regularized gravitational models can be set up in higher dimensions. Some analytic tools exist to study their state sums, but, unlike in $2 \mathrm{~d}$, no complete analytic solutions have yet been constructed. However, a
\end{abstract}


great advantage of our approach is the fact that it is well-suited for numerical simulations. In the second part of this review we describe the relevant Monte Carlo techniques, as well as some of the physical results that have been obtained from the simulations of Euclidean gravity. We also explain why the Lorentzian version of dynamical triangulations is a promising candidate for a non-perturbative theory of quantum gravity.

\section{Introduction}

There is at present no satisfactory theory of four-dimensional quantum gravity. This is partly related to the conceptual questions that arise when dealing with fluctuating geometries without reference to any background metric. Addressing these seems to call for a genuinely non-perturbative formulation of quantum gravity. The need for a non-perturbative approach persists in formulations where gravity appears embedded into a larger theory, such as string or M-theory. Although there have been attempts to identify non-perturbative structures within these unified theories (see $[1,2]$ and many successive papers), they so far seem to have raised as many questions as they have been able to answer. In these lectures we will review an alternative non-perturbative approach to quantum gravity. It is more conservative in spirit, in that it does not conjecture the existence of any radically new physical principles or symmetries. This so-called "dynamical triangulations" approach 1 can be formulated entirely within the framework of ordinary quantum field theory, while taking into account that the governing symmetry principle is reparametrization- (diffeomorphism-) invariance.

Our ansatz can be made in any dimension, and has been solved analytically in two space-time dimensions. In higher dimensions one so far has to rely largely on numerical simulations, and up to now only the case of Euclidean signature has been studied in detail. Although gravity in two dimensions has no genuine dynamical degrees of freedom, it does provide a testing ground for addressing some of the conceptual problems of quantum gravity explicitly, for example, how to define a notion of reparametrizationinvariant length in a fluctuating geometry or how to define correlation functions without reference to a background geometry. In 2d Euclidean gravity,

\footnotetext{
${ }^{1}$ The method of dynamical triangulations was introduced in the context of string theory and $2 \mathrm{~d}$ quantum gravity in $[3,4,5]$, and subsequently extended to higher-dimensional Euclidean quantum gravity $[6,7]$. An extensive review covering the developments up to 1996 can be found in the book [8]. A more recent summary is contained in [9], while the review [10] deals with a variety of lattice approaches to four-dimensional quantum gravity, including dynamical triangulations. The use of dynamical-triangulations methods in Lorentzian gravity was pioneered in $[11,12,13]$.
} 
a number of questions can be answered by continuum functional methods (within Liouville quantum field theory), but some of the most interesting problems involving geodesic distances can only be addressed in the nonperturbative setting of dynamical triangulations. The fact that in this latter approach quantum gravity is obtained as the scaling limit of a lattice theory shows that, contrary to common belief, discrete methods can be used also for reparametrization-invariant theories, as long as the discretization takes place directly on the space of geometries (that is, on the space of metrics modulo diffeomorphisms).

Another point that has been analyzed in the two-dimensional model is the analytic continuation between geometries of Lorentzian and Euclidean signature. Canonical quantization attempts of (Lorentzian) gravity usually take as their starting point globally hyperbolic manifolds equipped with a non-degenerate Lorentzian metric (which gives rise to a causal structure). However, it is a priori unclear to what extent these essentially classical structures should be preserved in the quantum theory. For example, in a covariant path-integral approach it is not obvious that a causality constraint should be imposed on each individual space-time configuration contributing to the amplitude. This idea was first advocated by Teitelboim [14], and more recently in $[15,16]$. One argument in its favour is that it seems hard to imagine how any notion of causality could emerge in the full quantum theory unless it had been imposed in some form on the individual histories in the first place.

Two-dimensional quantum gravity is an ideal testing ground for such ideas, since it can be solved explicitly. We will show that by restricting the state sum to discrete geometries with a causal structure one obtains a theory of quantum gravity with the following features $[11,12]$.

(1) The expectation values of reparametrization-invariant space-time distances have canonical dimensions. In other words, in spite of strong fluctuations in the geometry, there is still a sense in which the quantum space-time is two-dimensional.

(2) When matter with conformal charge $c \leq 1$ is coupled to this Lorentzian quantum gravity theory, both property (1) and the properties of the conformal field theory (critical exponents etc.) remain unchanged.

There is an alternative way of arriving at the same theory, namely, by starting from the geometric configurations of the $2 \mathrm{~d}$ Euclidean gravity theory, and removing its "baby universes" in a systematic manner. (The proliferation of these branching structures is responsible for most of the fractal and geometric properties of the Euclidean theory.) In this way one creates a many-to-1 correspondence between Euclidean and Lorentzian geometries. Lorentzian $2 \mathrm{~d}$ quantum gravity appears in this construction as a "renormalized" version of the Euclidean quantum theory [17]. 
Loosening the requirement of causality for the individual space-time histories, one may allow for changes in the topology of the spatial (constanttime) slices as a function of time, without changing the overall topology of space-time. This is of course tantamount to reintroducing geometries with baby universes. We will show below that once this process is permitted, it will totally dominate the structure of space-time. The resulting theory (which is equivalent to Euclidean Liouville quantum gravity) has the following features.

(1) The expectation values of geodesic space-time distances have anomalous dimensions, and the intrinsic Hausdorff dimension of quantum space-time is four, and not two [18, 19].

(2) When matter with conformal charge $c \leq 1$ is coupled to this Euclidean quantum gravity theory, both the fractal properties of the gravitational sector and the critical exponents of the conformal field theory are changed.

We therefore may say that the interaction between gravity and matter is weak in the Lorentzian $2 \mathrm{~d}$ gravity model, at least as long as $c \leq 1$. Allowing for baby-universe creation leads to a strong coupling between matter and gravity: the fractal properties of space-time become a function of the matter content and in turn the back-reaction of the fluctuating geometry changes the critical properties of the matter. The coupling of Ising models to $2 \mathrm{~d}$ gravity provides a particularly clear geometric illustration of the role played by the baby universes [20].

If one transcends the so-called $c=1$ barrier, even Lorentzian gravity exhibits a strong gravity-matter interaction, leading to a change in the fractal properties of space-time [13]. In the Euclidean case (i.e. including baby universes), it has been known for a long time that beyond $c=1$ the space-time disintegrates completely into fractals. These so-called branched polymers can be viewed as trees of baby universes of cut-off size. However, this is not what happens in the Lorentzian case. One does observe a phase transition for $c>1$ (to be precise, the transition must take place somewhere in the interval $1<c<4[13,21,22])$, but the new geometry is less pathological than the branched polymers. In particular, the critical matter exponents retain their Onsager values.

Many of the results quoted above for $c>1$ have been obtained by Monte Carlo simulations. Numerical methods have been very successful in the study of discretized quantum gravity. Moreover, in two-dimensional quantum gravity there has been a fruitful interaction between theory and "experiment". Non-rigorous calculations have been verified or falsified by numerical "experiments", and "observations" have inspired theoretical progress. In the Lorentzian case, visualizations of the computer-generated space-time 
geometries have been useful in understanding the influence of matter on geometry [23].

In higher dimensions, only partial results have been obtained by analytical methods. These include asymptotic estimates for the partition functions of dynamically triangulated Euclidean gravity in 3d and 4d [24, 25], and qualitative descriptions of the extreme branched-polymer and crumpled phases [25, 26, 27]. However, as already mentioned, our regularized quantum gravity models can readily be studied by means of computer simulations. In a first step, one determines the phase diagram of the discretized theory, in order to locate potential critical points where a continuum limit can be obtained. One can then use standard finite-size tools to study the scaling of various observables. This method has been very successful and has enabled us to study both the fractal properties of space-time, and the critical exponents of matter coupled to fluctuating geometries.

The rest of this paper is organized as follows. In Section 2.1 we describe and solve the simplest discretized Lorentzian model of $2 \mathrm{~d}$ gravity, using a

"Lorentzian" version of the method of dynamical triangulations. In Section 2.2, the corresponding continuum limit is obtained, while in Section 3 we demonstrate how the inclusion of baby universes changes the structure of quantum space-time. A non-perturbative definition of Euclidean quantum gravity for arbitrary dimension $d$ by means of dynamical triangulations is given in Section 1 , where we also discuss the inclusion of matter fields. Section 5 outlines the principles for numerical simulations of the model, with special emphasis on $2 \mathrm{~d}$ gravity. In Subsection 5.2, we describe various possibilities of defining notions of (fractal) "dimensionality" in the framework of Euclidean quantum gravity. We discuss why these "observables" are particularly well-suited for use in numerical calculations. Subsection 5.3 provides the interpretation of the numerical results obtained in $2 \mathrm{~d} \mathrm{Eu-}$ clidean quantum gravity. Section 6 describes the numerical approach to higher-dimensional Euclidean quantum gravity. Finally, in Section 7, we outline the future perspectives for both Euclidean and Lorentzian lattice gravity.

\section{Lorentzian gravity in $2 d$}

\subsection{THE DISCRETE MODEL}

In order to solve two-dimensional quantum gravity in a path-integral formulation, one has to "count" geometries. Since for a fixed space-time topology the gravitational action consists only of the cosmological term, all geome- 
tries of a fixed space-time volume contribute with the same weight 2 . In the simplest model of two-dimensional gravity, no spatial topology changes are allowed either. For simplicity, we choose the spatial slices (at constant "time") to be circles, so that the overall topology of space-time is of the form $S^{1} \times[0,1]$ (for other spatial boundary conditions, see [28]). This mimicks the situation in classical gravity, where one usually works with globally hyperbolic space-times [29]. We will use a natural class of triangulations of the cylinder $S^{1} \times[0,1]$, to which we will assign edge lengths (i.e. a discretized metric) in such a way that each two-dimensional geometry carries a discrete causal structure. Our simplicial space-times will have a preferred foliation into a discrete set of circular slices, consisting only of space-like edges. The foliation parameter $t$ can be interpreted as a discrete version of "proper time". The main task of our dynamical-triangulations approach is to count the geometries in this class, that is, to perform the state sum in the regularized context, and then attempt to take a continuum limit.

The geometry of each spatial slice is uniquely characterized by the length assigned to it. In the discretized version, the length $L$ will be "quantized" in units of a lattice spacing $a$, i.e. $L=l \cdot a$ where $l$ is an integer. A slice will thus be defined by $l$ vertices and $l$ links connecting them. To obtain a $2 \mathrm{~d}$ geometry, we will evolve this spatial loop in discrete steps. This leads to a preferred notion of (discrete) "time" $t$, where each loop represents a slice of constant $t$. The propagation from time-slice $t$ to time-slice $t+1$ is governed by the following rule: each vertex $i$ at time $t$ is connected to $k_{i}$ vertices at time $t+1, k_{i} \geq 1$, by links which are assigned squared edge lengths $-a^{2}$. The $k_{i}$ vertices, $k_{i}>1$, at time-slice $t+1$ will be connected by $k_{i}-1$ consecutive space-like links, thus forming $k_{i}-1$ triangles. Finally the right boundary vertex in the set of $k_{i}$ vertices will be identified with the left boundary vertex of the set of $k_{i+1}$ vertices. In this way we get a total of $\sum_{i=1}^{l}\left(k_{i}-1\right)$ vertices (and also links) at time-slice $t+1$ and the two spatial slices are connected by $\sum_{i=1}^{l} k_{i} \equiv l_{t}+l_{t+1}$ triangles (see Fig. 1).

The elementary building blocks of a geometry are therefore triangles with one space- and two time-like edges. We define them to be flat in the interior. A consistent way of assigning interior angles to such Minkowskian triangles is described in [30]. The angle between two time-like edges is $\gamma_{t t}=$ $-\arccos \frac{3}{2}$, and between a space- and a time-like edge $\gamma_{s t}=\frac{\pi}{2}+\frac{1}{2} \arccos \frac{3}{2}$, summing up to $\gamma_{t t}+2 \gamma_{s t}=\pi$. The sum over all angles around a vertex with $j$ incoming and $k$ outgoing time-like edges (by definition $j, k \geq 1$ ) is given by $2 \pi+(4-j-k) \arccos \frac{3}{2}$. The regular triangulation of flat Minkowski space corresponds to $j=k=2$ at all vertices. The volume of a single triangle is given by $\frac{\sqrt{5}}{4} a^{2}$.

\footnotetext{
${ }^{2}$ Maybe surprisingly, in the framework of dynamical triangulations also higherdimensional gravity can be reduced to a pure counting problem, c.f. Section 4.3 .
} 


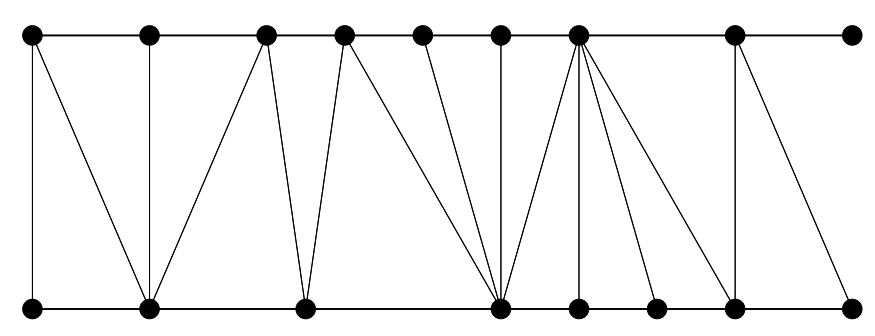

Figure 1. The propagation of a spatial slice from step $t$ to step $t+1$. The ends of the strip should be joined to form a band with topology $S^{1} \times[0,1]$.

One may view these geometries as a subclass of all possible triangulations that allow for the introduction of a causal structure. Namely, if we think of all time-like links as being future-directed, a vertex $v^{\prime}$ lies in the future of a vertex $v$ iff there is an oriented sequence of time-like links leading from $v$ to $v^{\prime}$. Two arbitrary vertices may or may not be causally related in this way.

In quantum gravity we are instructed to sum over all geometries connecting, say, two spatial boundaries of length $L_{1}$ and $L_{2}$, with the weight of each geometry $g$ given by

$$
\mathrm{e}^{i S[g]}, \quad S[g]=\Lambda \int \sqrt{-\operatorname{det} g} \quad(\text { in } 2 \mathrm{~d}),
$$

where $\Lambda$ is the cosmological constant. In our discretized model the boundaries will be characterized by integers $l_{1}$ and $l_{2}$, the number of vertices or links at the two boundaries. The path-integral amplitude for the propagation from geometry $l_{1}$ to $l_{2}$ will be the sum over all interpolating surfaces of the kind described above, with a weight given by the discretized version of (1). Let us call the corresponding amplitude $G_{\lambda}^{(1)}\left(l_{1}, l_{2}\right)$. We thus have

$$
\begin{aligned}
G_{\lambda}^{(1)}\left(l_{1}, l_{2}\right) & =\sum_{t=1}^{\infty} G_{\lambda}^{(1)}\left(l_{1}, l_{2} ; t\right), \\
G_{\lambda}^{(1)}\left(l_{1}, l_{2} ; t\right) & =\sum_{l=1}^{\infty} G_{\lambda}^{(1)}\left(l_{1}, l ; 1\right) l G_{\lambda}^{(1)}\left(l, l_{2}, t-1\right), \\
G_{\lambda}^{(1)}\left(l_{1}, l_{2} ; 1\right) & =\frac{1}{l_{1}} \sum_{\left\{k_{1}, \ldots, k_{l_{1}}\right\}} \mathrm{e}^{i \lambda a^{2} \sum_{i=1}^{l_{1}} k_{i}},
\end{aligned}
$$

where $\lambda$ denotes the bare cosmological constant (we have absorbed the finite triangle volume factor), and where $t$ denotes the total number of time-slices connecting $l_{1}$ and $l_{2}$.

\footnotetext{
${ }^{3}$ One obtains the renormalized (continuum) cosmological constant $\Lambda$ in (1) by an additive renormalization, see below.
} 
From a combinatorial point of view it is convenient to mark a vertex on the entrance loop in order to get rid of the factors $l$ and $1 / l$ in (3) and (4), that is,

$$
G_{\lambda}\left(l_{1}, l_{2} ; t\right) \equiv l_{1} G_{\lambda}^{(1)}\left(l_{1}, l_{2} ; t\right)
$$

(the unmarking of a point may be thought of as the factoring out by (discrete) spatial diffeomorphisms). Note that $G_{\lambda}\left(l_{1}, l_{2} ; 1\right)$ plays the role of a transfer matrix, satisfying

$$
\begin{aligned}
G_{\lambda}\left(l_{1}, l_{2} ; t_{1}+t_{2}\right) & =\sum_{l} G_{\lambda}\left(l_{1}, l ; t_{1}\right) G_{\lambda}\left(l, l_{2} ; t_{2}\right) \\
G_{\lambda}\left(l_{1}, l_{2} ; t+1\right) & =\sum_{l} G_{\lambda}\left(l_{1}, l ; 1\right) G_{\lambda}\left(l, l_{2} ; t\right) .
\end{aligned}
$$

Knowing $G_{\lambda}\left(l_{1}, l_{2} ; 1\right)$ allows us to find $G_{\lambda}\left(l_{1}, l_{2} ; t\right)$ by iterating (17) $t$ times. This program is conveniently carried out by introducing the generating function for the numbers $G_{\lambda}\left(l_{1}, l_{2} ; t\right)$,

$$
G_{\lambda}(x, y ; t) \equiv \sum_{k, l} x^{k} y^{l} G_{\lambda}(k, l ; t)
$$

which we can use to rewrite (16) as

$$
G_{\lambda}\left(x, y ; t_{1}+t_{2}\right)=\oint \frac{d z}{2 \pi i z} G_{\lambda}\left(x, z^{-1} ; t_{1}\right) G_{\lambda}\left(z, y ; t_{2}\right),
$$

where the contour should be chosen to include the singularities in the complex $z$-plane of $G_{\lambda}\left(x, z^{-1} ; t_{1}\right)$ but not those of $G_{\lambda}\left(z, y ; t_{2}\right)$.

One can either view the introduction of $G_{\lambda}(x, y ; t)$ as a purely technical device or take $x$ and $y$ as boundary cosmological constants,

$$
x=\mathrm{e}^{i \lambda_{i} a}, \quad y=\mathrm{e}^{i \lambda_{o} a},
$$

such that $x^{k}=\mathrm{e}^{i \lambda_{i} a k}$ becomes a boundary cosmological term, and similarly for $y^{l}=\mathrm{e}^{i \lambda_{o} a l}$. Let us for notational convenience define

$$
g=\mathrm{e}^{i \lambda a^{2}}
$$

(not to be confused with the symbol for the continuum metric). For the technical purpose of counting we view $x, y$ and $g$ as variables in the complex plane. In general the function

$$
G(x, y ; g ; t) \equiv G_{\lambda}(x, y ; t)
$$

will be analytic in a neighbourhood of $(x, y, g)=(0,0,0)$. 
From the definitions (4) and (5) it follows by standard techniques of generating functions that we may associate a factor $g$ with each triangle, a factor $x$ with each vertex on the entrance loop and a factor $y$ with each vertex on the exit loop, leading to

$$
G(x, y ; g ; 1)=\sum_{k=0}^{\infty}\left(g x \sum_{l=0}^{\infty}(g y)^{l}\right)^{k}-\sum_{k=0}^{\infty}(g x)^{k}=\frac{g^{2} x y}{(1-g x)(1-g x-g y)} .
$$

Formula (13) is simply a book-keeping device for all possible ways of evolving from an entrance loop of any length in one step to an exit loop of any length. The subtraction of the term $1 /(1-g x)$ has been performed to exclude the degenerate cases where either the entrance or the exit loop is of length zero.

From (13) and eq. (9), with $t_{1}=1$, we obtain

$$
G(x, y ; g ; t)=\frac{g x}{1-g x} G\left(\frac{g}{1-g x}, y ; g ; t-1\right) .
$$

This equation can be iterated and the solution written as

$$
G(x, y ; g ; t)=F_{1}^{2}(x) F_{2}^{2}(x) \cdots F_{t-1}^{2}(x) \frac{g^{2} x y}{\left[1-g F_{t-1}(x)\right]\left[1-g F_{t-1}(x)-g y\right]},
$$

where $F_{t}(x)$ is defined iteratively by

$$
F_{t}(x)=\frac{g}{1-g F_{t-1}(x)}, \quad F_{0}(x)=x .
$$

Let $F$ denote the fixed point of this iterative equation. By standard techniques one readily obtains

$$
F_{t}(x)=F \frac{1-x F+F^{2 t-1}(x-F)}{1-x F+F^{2 t+1}(x-F)}, \quad F=\frac{1-\sqrt{1-4 g^{2}}}{2 g} .
$$

Inserting (17) in eq. (15), we can write

$$
\begin{aligned}
& G(x, y ; g, t)=\frac{F^{2 t}\left(1-F^{2}\right)^{2} x y}{\left(A_{t}-B_{t} x\right)\left(A_{t}-B_{t}(x+y)+C_{t} x y\right)} \\
& \quad=\frac{F^{2 t}\left(1-F^{2}\right)^{2} x y}{\left[(1-x F)-F^{2 t+1}(F-x)\right]\left[(1-x F)(1-y F)-F^{2 t}(F-x)(F-y)\right]}
\end{aligned}
$$

where the time-dependent coefficients are given by

$$
A_{t}=1-F^{2 t+2}, \quad B_{t}=F\left(1-F^{2 t}\right), \quad C_{t}=F^{2}\left(1-F^{2 t-2}\right) .
$$


The combined region of convergence to the expansion in powers $g^{k} x^{l} y^{m}$, valid for all $t$ is

$$
|g|<\frac{1}{2}, \quad|x|<1, \quad|y|<1 .
$$

\subsection{THE CONTINUUM LIMIT}

The path integral formalism we are using here is very similar to the one used to represent the free particle as a sum over paths. Also there one performs a summation over geometric objects (the paths), and the path integral itself serves as the propagator. From the particle case it is known that the bare mass undergoes an additive renormalization (even for the free particle), and that the bare propagator is subject to a wave-function renormalization (see [8] for a review). The same is true in two-dimensional Euclidean gravity, treated in the formalism of dynamical triangulations [8]. The coupling constants with positive mass dimension, i.e. the cosmological constant and the boundary cosmological constants, undergo an additive renormalization, while the partition function itself (i.e. the Hartle-Hawking-like wave function) undergoes a multiplicative wave-function renormalization. We therefore expect the bare coupling constants $\lambda, \lambda_{i}$ and $\lambda_{0}$ to behave as

$$
\lambda=\frac{C_{\lambda}}{a^{2}}+\tilde{\Lambda}, \quad \lambda_{i}=\frac{C_{\lambda_{i}}}{a}+\tilde{X}, \quad \lambda_{o}=\frac{C_{\lambda_{o}}}{a}+\tilde{Y},
$$

where $\tilde{\Lambda}, \tilde{X}, \tilde{Y}$ denote the renormalized cosmological and boundary cosmological constants. If we introduce the notation

$$
g_{c}=\mathrm{e}^{i C_{\lambda}}, \quad x_{c}=\mathrm{e}^{i C_{\lambda_{i}}}, \quad y_{c}=\mathrm{e}^{i C_{\lambda_{o}}},
$$

for critical values of the coupling constants, it follows from (10) and (11) that

$$
g=g_{c} \mathrm{e}^{i a^{2} \tilde{\Lambda}}, \quad x=x_{c} \mathrm{e}^{i a \tilde{X}}, \quad y=y_{c} \mathrm{e}^{i a \tilde{Y}} .
$$

The wave-function renormalization will appear as a multiplicative cut-off dependent factor in front of the bare "Green's function" $G(x, y ; g ; t)$,

$$
G_{\tilde{\Lambda}}(\tilde{X}, \tilde{Y} ; T)=\lim _{a \rightarrow 0} a^{\eta} G(x, y ; g ; t),
$$

where $T=a t$, and where the critical exponent $\eta$ should be chosen so that the right-hand side of eq. (25) exists. In general this will only be possible for particular choices of $g_{c}, x_{c}$ and $y_{c}$ in (25).

The basic relation (6) can survive the limit (25) only if $\eta=1$, since we have assumed that the boundary lengths $L_{1}$ and $L_{2}$ have canonical dimensions and satisfy $L_{i}=a l_{i}$. 
A closer analysis reveals that only at $g_{c}=1 / 2$ one can obtain a sensible continuum limit. It corresponds to a purely imaginary bare cosmological constant $\lambda_{c}:=C_{\lambda} / a^{2}=-i \ln 2 / a^{2}$. If we want to approach this point from the region in the complex $g$-plane where $G(x, y ; g ; t)$ converges it is natural to choose the renormalized coupling $\tilde{\Lambda}$ imaginary as well, $\tilde{\Lambda}=i \Lambda$, i.e.

$$
\lambda=i \frac{\ln \frac{1}{2}}{a^{2}}+i \Lambda \text {. }
$$

One obtains a well-defined scaling limit (corresponding to $\Lambda \in \mathbb{R}$ ) by letting $\lambda \rightarrow \lambda_{c}$ along the imaginary axis. The Lorentzian form for the continuum propagator is obtained by an analytic continuation $\Lambda \rightarrow-i \Lambda$ in the renormalized coupling of the resulting Euclidean expressions.

At this stage it may seem that we are surreptitiously reverting to a fully Euclidean model. We could of course equivalently have conducted the entire discussion up to this point in the "Euclidean sector", by omitting the factor of $-i$ in the exponential (1) of the action, choosing $\lambda$ positive real and taking all edge lengths equal to 1 . However, from a purely Euclidean point of view there would not have been any reason for restricting the state sum to a subclass of geometries admitting a causal structure. The associated preferred notion of a discrete "time" allows us to define an "analytic continuation in time". Because of the simple form of the action in two dimensions, the rotation

$$
\int d x d t \sqrt{-\operatorname{det} g_{l o r}} \rightarrow i \int d x d t_{e u} \sqrt{\operatorname{det} g_{e u}}
$$

to Euclidean metrics in our model is equivalent to the analytic continuation of the cosmological constant $\Lambda$.

From (18) or (19) it follows that we can only get macroscopic loops in the limit $a \rightarrow 0$ if we simultaneously take $x, y \rightarrow 1$. (For $g_{c}=-1 / 2$, one needs to take $x, y \rightarrow-1$. The continuum expressions one obtains are identical to those for $g_{c}=1 / 2$.) Again the critical points correspond to purely imaginary bare boundary cosmological coupling constants. We will allow for such imaginary couplings and thus approach the critical point $\lambda_{i}=\lambda_{o}=0$ from the region of convergence of $G(x, y ; g ; t)$, i.e. via real, positive $X, Y$ where

$$
\lambda_{i}=i X a, \quad \lambda_{o}=i Y a .
$$

Again $X$ and $Y$ have an obvious interpretation as positive boundary cosmological constants in a Euclidean theory, which may be analytically continued to imaginary values to reach the Lorentzian sector.

Summarizing, we have

$$
g=\frac{1}{2} \mathrm{e}^{-\Lambda a^{2}} \rightarrow \frac{1}{2}\left(1-\Lambda a^{2}\right), \quad(\text { i.e. } \quad F=1-a \sqrt{\Lambda})
$$


as well as

$$
x=\mathrm{e}^{-X a} \rightarrow 1-a X, \quad y=\mathrm{e}^{-a Y} \rightarrow 1-a Y,
$$

where the arrows $\rightarrow$ in $(29)$ and $(30)$ should be viewed as analytic coupling constant redefinitions of $\Lambda, X$ and $Y$, which we have performed to get rid of factors of $1 / 2$ etc. in the formulas below. With the definitions (29) and (30) it is straightforward to perform the continuum limit of $G(x, y ; g ; t)$ as $(x, y, g) \rightarrow\left(x_{c}, y_{c}, g_{c}\right)=(1,1,1 / 2)$, yielding

$$
\begin{aligned}
& G_{\Lambda}(X, Y ; T)=\frac{4 \Lambda \mathrm{e}^{-2 \sqrt{\Lambda} T}}{(\sqrt{\Lambda}+X)+\mathrm{e}^{-2 \sqrt{\Lambda} T}(\sqrt{\Lambda}-X)} \\
& \quad \times \frac{1}{(\sqrt{\Lambda}+X)(\sqrt{\Lambda}+Y)-\mathrm{e}^{-2 \sqrt{\Lambda} T}(\sqrt{\Lambda}-X)(\sqrt{\Lambda}-Y)} .
\end{aligned}
$$

For $T \rightarrow \infty$ one finds

$$
G_{\Lambda}(X, Y ; T) \stackrel{T \rightarrow \infty}{\longrightarrow} \frac{4 \Lambda \mathrm{e}^{-2 \sqrt{\Lambda} T}}{(X+\sqrt{\Lambda})^{2}(Y+\sqrt{\Lambda})},
$$

while the limit for $T \rightarrow 0$ is

$$
G_{\Lambda}(X, Y ; T=0)=\frac{1}{X+Y},
$$

in accordance with the expectation that

$$
G_{\Lambda}\left(L_{1}, L_{2} ; T=0\right)=\delta\left(L_{1}-L_{2}\right) .
$$

The general expression for $G_{\Lambda}\left(L_{1}, L_{2} ; T\right)$ can be computed as the inverse Laplace transform of formula (31), yielding

$$
G_{\Lambda}\left(L_{1}, L_{2} ; T\right)=\frac{\mathrm{e}^{-[\operatorname{coth} \sqrt{\Lambda} T] \sqrt{\Lambda}\left(L_{1}+L_{2}\right)}}{\sinh \sqrt{\Lambda} T} \frac{\sqrt{\Lambda L_{1} L_{2}}}{L_{2}} I_{1}\left(\frac{2 \sqrt{\Lambda L_{1} L_{2}}}{\sinh \sqrt{\Lambda} T}\right),
$$

where $I_{1}(x)$ is a modified Bessel function of the first kind. The asymmetry between $L_{1}$ and $L_{2}$ arises because the entrance loop has a marked point, whereas the exit loop has not. The amplitude with both loops marked is obtained by multiplying with $L_{2}$, while the amplitude with no marked loops is obtained after dividing (35) by $L_{1}$. Quite remarkably, our highly non-trivial expression (35) agrees with the loop propagator obtained from a bona-fide continuum calculation in proper-time gauge of pure $2 \mathrm{~d}$ gravity by Nakayama [31].

The basic result (31) for $G_{\Lambda}(X, Y ; T)$ can be derived by taking the continuum limit of the recursion relation (14). By inserting (29) and (30) in eq. (14) and expanding to first order in the lattice spacing $a$ we obtain

$$
\frac{\partial}{\partial T} G_{\Lambda}(X, Y ; T)+\frac{\partial}{\partial X}\left[\left(X^{2}-\Lambda\right) G_{\Lambda}(X, Y ; T)\right]=0 .
$$


This is a standard first-order partial differential equation which should be solved with the boundary condition $(33)$ at $T=0$, since this expresses the natural condition (34) on $G_{\Lambda}\left(L_{1}, L_{2}\right)$. The solution is thus

$$
G_{\Lambda}(X, Y ; T)=\frac{\bar{X}^{2}(T ; X)-\Lambda}{X^{2}-\Lambda} \frac{1}{\bar{X}(T ; X)+Y},
$$

where $\bar{X}(T ; X)$ is the solution to the characteristic equation

$$
\frac{d \bar{X}}{d T}=-\left(\bar{X}^{2}-\Lambda\right), \quad \bar{X}(T=0)=X .
$$

It is readily seen that the solution is indeed given by (31) since we obtain

$$
\bar{X}(T)=\sqrt{\Lambda} \frac{(\sqrt{\Lambda}+X)-\mathrm{e}^{-2 \sqrt{\Lambda} T}(\sqrt{\Lambda}-X)}{(\sqrt{\Lambda}+X)+\mathrm{e}^{-2 \sqrt{\Lambda}} T(\sqrt{\Lambda}-X)} .
$$

If we interpret the propagator $G_{\Lambda}\left(L_{1}, L_{2} ; T\right)$ as the matrix element between two boundary states of a Hamiltonian evolution in "time" $T$,

$$
G_{\Lambda}\left(L_{1}, L_{2} ; T\right)=\left\langle L_{1}\left|\mathrm{e}^{-\hat{H} T}\right| L_{2}\right\rangle
$$

we can, after an inverse Laplace transformation, read off the functional form of the Hamiltonian operator $\hat{H}$ from (36),

$$
\hat{H}\left(L, \frac{\partial}{\partial L}\right)=-L \frac{\partial^{2}}{\partial L^{2}}+L \Lambda .
$$

The corresponding Hamiltonian for the propagator of unmarked loops is given by

$$
\hat{H}_{u}\left(L, \frac{\partial}{\partial L}\right)=-L \frac{\partial^{2}}{\partial L^{2}}-2 \frac{\partial}{\partial L}+\Lambda L .
$$

The Hamiltonian (42) is Hermitian with respect to the natural measure $L \mathrm{~d} L$, which has its origin in the basic completeness relation (3) for the transfer matrix with unmarked entrance and exit loops. If one wants to construct a unitary evolution with respect to the "time"-parameter $T$ appearing in the transfer-matrix approach, one can simply exponentiate $(i \hat{H} T)$.

However, we should point out that there is an alternative to the analytic continuation $T \rightarrow-i T$ if one wants to relate the Euclidean and Lorentzian sectors of the theory. The combination $\sqrt{\Lambda} T$ appearing as an argument in (35) arises in taking the continuum limit of powers of the form $F^{t}$ in expressions like (18), (19), where $F$ is defined in (17). There are two aspects to a possible analytic continuation of $F^{t}$. The power $t$ in $F^{t}$ should clearly not be 
continued, since it is simply an integer counting the number of iterations of the transfer matrix. However, the function $F$ itself does refer to the action, because the dimensionless coupling constant $g=\mathrm{e}^{i \lambda a_{t} a_{l}}$ is the action for a single Lorentzian triangle. (For added clarity we have distinguished between the lattice spacings in time- and space-directions, and called them $a_{t}$ and $a_{l}$.) From the expression for $F$ in terms of $g$ in (17), we have $F=1-\sqrt{a_{t} a_{l} \Lambda}$. The analytic continuation of $F$ in time, from Euclidean to Lorentzian time, corresponds to the substitution $a_{t} \rightarrow-i a_{t}$ under the square-root sign, and thus becomes equivalent to the continuation $\Lambda \rightarrow-i \Lambda$ in the cosmological constant, as already remarked below eq. (27). The subtleties associated with the analytic continuation in the "time"-parameter $T$ appearing in a transfer-matrix formulation of quantum gravity were first discussed in $[32,33]$ in the context of a square-root action formulation. Similar difficulties will also be present in higher-dimensional gravity, where the analytic continuation from Euclidean metrics to Lorentzian metrics cannot be absorbed by a continuation in $\Lambda$ alone. To conclude, it is not obvious how to choose the correct analytic continuation back to Lorentzian signature, once the continuum limit has been taken. The continuation $T \rightarrow-i T$ leads to a unitary theory. For the continuation $\Lambda \rightarrow-i \Lambda$, we have not yet found a scalar product which makes the corresponding evolution operator unitary.

\section{Topology changes and Euclidean quantum gravity}

\subsection{BABY UNIVERSE CREATION}

In our non-perturbative regularization of $2 \mathrm{~d}$ quantum gravity we have so far not included the possibility of topology changes of space. We will now show that if one allows for spatial topology changes, one is led in an essentially unambiguous manner to a different theory of two-dimensional quantum gravity, where space-time is much more fractal, and which agrees with Euclidean 2d quantum gravity as defined by Euclidean dynamical triangulations or Liouville theory.

By a topology change of space in our Lorentzian setting we have in mind the following: a baby universe may branch off at some time $T$ and develop in the future, where it will eventually disappear in the vacuum, but it is not allowed to rejoin the "parent" universe and thus change the overall topology of the two-dimensional manifold. This is a restriction we impose to be able to compare with the analogous calculation in usual $2 \mathrm{~d}$ Euclidean quantum gravity.

It is well known that such a branching leads to additional complications, compared with the Euclidean situation, in the sense that, in general, no continuum Lorentzian metrics which are smooth and non-degenerate everywhere can be defined on such space-times (see, for example, [34] and ref- 
erences therein). These considerations do not affect the cosmological term in the action, but lead potentially to contributions from the Einstein-Hilbert term at the singular points where a branching or pinching occurs.

We have so far ignored the curvature term in the action since it gives merely a constant contribution in the absence of topology change. We will continue to do so in the slightly generalized setting just introduced. The continuum results of [34] suggest that the contributions from the two singular points associated with each branching of a baby universe (one at the branching point and one at the tip of the baby universe where it contracts to a point) cancel in the action. The physical geometry of these configurations may seem slightly contrived, but they may well be important in the quantum theory of gravity and deserve further study. However, for the moment our main motivation for introducing them is to make contact with the usual non-perturbative Euclidean path-integral results.

We will use the rest of this section to demonstrate the following: once we allow for spatial topology changes,

(1) this process completely dominates and changes the critical behaviour of the discretized theory, and

(2) the disc amplitude $W_{\Lambda}(L)$ (the Hartle-Hawking wave function) is uniquely determined, almost without any calculations.

Our starting point will be the discretized model introduced in Sec. 2.1. In this model we do not directly have a disc amplitude like the Hartle-Hawking wave functional. However, as discussed at the beginning of this section, the degeneracy of the metric at a point in the interior of the disc is always compensated (in the sense of complex contributions to the action) by the degeneracy of the metric at the point where the baby universe branched off. We can thus define the disc amplitude in Lorentzian gravity as

$$
w^{(b)}(x, g):=\sum_{t} G^{(b)}\left(x, l_{2}=1 ; g ; t\right)=G^{(b)}\left(x, l_{2}=1 ; g\right),
$$

and the continuum version as

$$
W_{\Lambda}^{(b)}(X)=\int \mathrm{d} T G_{\Lambda}^{(b)}\left(X, L_{2}=0 ; T\right)=G_{\Lambda}^{(b)}\left(X, L_{2}=0\right)
$$

where the superscript ${ }^{(b)}$ indicates the "bare" Lorentzian model without spatial topology changes. It follows that

$$
w^{(b)}(x, g) \rightarrow a^{-1} W_{\Lambda}^{(b)}(X) .
$$

There are a number of ways to implement the creation of baby universes, some more natural than others, but they all agree in the continuum limit, as will be clear from the general arguments provided below. Here we discuss 


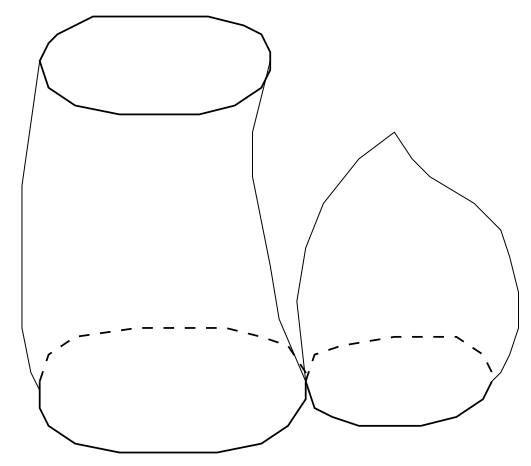

Figure 2. A "baby universe" created by a global pinching.

only the simplest way of implementing such a change. This is shown in Fig. 2: stepping forward from $t$ to $t+1$ from a loop of length $l_{1}$ we create a baby universe of length $l<l_{1}$ by pinching it off non-locally from the main branch.

Accounting for the new possibilities of evolution in each step according to fig. 2, the new and old transfer matrices are related by

$$
G_{\lambda}\left(l_{1}, l_{2} ; 1\right)=G_{\lambda}^{(b)}\left(l_{1}, l_{2} ; 1\right)+\sum_{l=1}^{l_{1}-1} l_{1} w\left(l_{1}-l, g\right) G_{\lambda}^{(b)}\left(l, l_{2} ; 1\right) .
$$

The factor $l_{1}$ in the sum comes from the fact that the "pinching" shown in fig. 2 can take place at any of the $l_{1}$ vertices. As before, the new transfer matrix leads to new amplitudes $G_{\lambda}\left(l_{1}, l_{2} ; t\right)$, satisfying

$$
G_{\lambda}\left(l_{1}, l_{2} ; t_{1}+t_{2}\right)=\sum_{l} G_{\lambda}\left(l_{1}, l ; t_{1}\right) G_{\lambda}\left(l, l_{2} ; t_{2}\right),
$$

and in particular

$$
G_{\lambda}\left(l_{1}, l_{2} ; t\right)=\sum_{l} G_{\lambda}\left(l_{1}, l ; 1\right) G_{\lambda}\left(l, l_{2} ; t-1\right) .
$$

Performing a (discrete) Laplace transformation of eq. (48) leads to

$$
\begin{aligned}
& G(x, y ; g ; t)= \\
& \quad \oint \frac{d z}{2 \pi i z}\left[G_{\lambda}^{(b)}\left(x, z^{-1} ; 1\right)+x \frac{\partial}{\partial x}\left(w(x ; g) G_{\lambda}^{(b)}\left(x, z^{-1} ; 1\right)\right)\right] G(z, y ; g ; t-1),
\end{aligned}
$$

or, using the explicit form of the transfer matrix $G_{\lambda}^{(b)}(x, z ; 1)$, formula (13),

$$
G(x, y ; g ; t)=\left[1+x \frac{\partial w(x, g)}{\partial x}+x w(x, g) \frac{\partial}{\partial x}\right] \frac{g x}{1-g x} G\left(\frac{g}{1-g x}, y ; g ; t-1\right) \text {. }
$$


At this point neither the disc amplitude $w(x, g)$ nor $G(x, y ; g ; t)$ are known. We will now show that they are uniquely determined if we assume that the boundary length scales canonically with the lattice spacing, $L=a l$, implying a renormalized boundary cosmological constant $X$ with the dimension of mass, $x=x_{c}(1-a X)$. In addition we assume that the dimension of the renormalized cosmological constant $\Lambda$ is canonical too, $g=g_{c}\left(1-\frac{1}{2} \Lambda a^{2}\right)$. Somewhat related arguments have been presented in different settings in $[35,36]$.

It follows from relation (47) that we need

$$
G_{\lambda}\left(l_{1}, l_{2}, t\right) \stackrel{a \rightarrow 0}{\longrightarrow} a G_{\Lambda}\left(L_{1}, L_{2} ; T\right) .
$$

It is important for the following discussion that $G_{\lambda}\left(l_{1}, l_{2} ; t\right)$ cannot contain a non-scaling part since from first principles (sub-additivity) it has to decay exponentially in $t$. By a Laplace transformation, using $x=x_{c}(1-a X)$ in the scaling limit, we thus conclude that

$$
G_{\lambda}\left(x, l_{2}, t\right) \stackrel{a \rightarrow 0}{\longrightarrow} G_{\Lambda}\left(X, L_{2}, T\right),
$$

and further, by a Laplace transformation in $L_{2}$,

$$
G_{\lambda}(x, y ; t) \stackrel{a \rightarrow 0}{\longrightarrow} a^{-1} G_{\Lambda}(X, Y ; T) .
$$

We will now show that the scaling of $w(x, g)$ is quite restricted too. The starting point is a combinatorial identity which the disc amplitude has to satisfy. The arguments are valid both for the disc amplitude in Euclidean quantum gravity and the disc amplitude we have introduced for our model in (43). We will assume the general scaling

$$
w(x, g)=w_{n s}(x, g)+a^{\eta} W_{\Lambda}(X)+\text { less singular terms }
$$

for the disc amplitude. In the case $\eta<0$ the first term is considered absent (or irrelevant). However, if $\eta>0$ a term like $w_{n s}$ will generically be present, since any slight redefinition of the coupling constants of the model will produce such a term if it was not there from the beginning.

We will introduce an explicit mark in the bulk of $w(x, g)$ by differentiating with respect to $g$. This leads to the combinatorial identity

$$
g \frac{\partial w(x, g)}{\partial g}=\sum_{t} \sum_{l} G(x, l ; g ; t) l w(l, g)
$$

or, after the usual Laplace transform,

$$
g \frac{\partial w(x, g)}{\partial g}=\sum_{t} \oint \frac{d z}{2 \pi i z} G\left(x, z^{-1} ; g ; t\right) \frac{\partial w(z, g)}{\partial z} .
$$




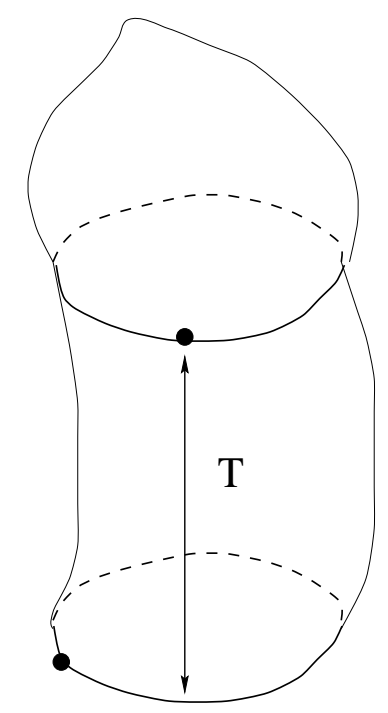

Figure 3. Marking a vertex in the bulk of $W_{\Lambda}(X)$. The mark has a distance $T$ from the boundary loop, which itself has one marked vertex.

The situation is illustrated in Fig. 3. A given mark has a distance $t$ ( $T$ in the continuum) to the entrance loop. In the figure we have drawn all points which have the same distance to the entrance loop and which form a connected loop. In the bare model these are all the points at distance $t$. In the case where baby universes are allowed (which we have not included in the figure), there can be many disconnected loops at the same distance. Let us assume a general scaling

$$
T=a^{\varepsilon} t, \quad \varepsilon>0,
$$

for the time variable $T$ in the continuum limit. Above we saw that the bare model without baby universe creation corresponded to $\varepsilon=1$. With the generalization (57) we account for the fact that by allowing for baby universes we have introduced an explicit asymmetry between the time- and space-directions.

Inserting (54) and (57) into eq. (56) we obtain

$$
\begin{aligned}
& \frac{\partial w_{n s}}{\partial g}-2 a^{\eta-2} \frac{\partial W_{\Lambda}(X)}{\partial \Lambda}= \\
& \frac{1}{a^{\varepsilon}} \int \mathrm{d} T \int d Z G_{\Lambda}(X,-Z ; T)\left[\frac{\partial w_{n s}}{\partial z}-a^{\eta-1} \frac{1}{z_{c}} \frac{\partial W_{\Lambda}(Z)}{\partial Z}\right],
\end{aligned}
$$

where $(x, g)=\left(x_{c}, g_{c}\right)$ in the non-singular part.

From eq. (58) and the requirement $\epsilon>0$ it follows that the only consistent choices for $\eta$ are 
1: $\boldsymbol{\eta}<0$, i.e.

$$
a^{\eta-2} \frac{\partial W_{\Lambda}(X)}{\partial \Lambda}=\frac{a^{\eta-1}}{2 a^{\varepsilon}} \int \mathrm{d} T \int d Z G_{\Lambda}(X,-Z ; T) \frac{1}{z_{c}} \frac{\partial W_{\Lambda}(Z)}{\partial Z},
$$

in which case we get $\varepsilon=1$; and

2: $\mathbf{1}<\boldsymbol{\eta}<\mathbf{2}$. Here formula (58) splits into the two equations

$$
-a^{\eta-2} \frac{\partial W_{\Lambda}(X)}{\partial \Lambda}=\left.\frac{1}{2 a^{\varepsilon}} \frac{\partial w_{n s}}{\partial z}\right|_{z=x_{c}} \int \mathrm{d} T \int d Z G_{\Lambda}(X,-Z ; T),
$$

and

$$
\left.\frac{\partial w_{n s}}{\partial g}\right|_{g=g_{c}}=-\frac{a^{\eta-1}}{a^{\varepsilon}} \int \mathrm{d} T \int d Z G_{\Lambda}(X,-Z ; T) \frac{1}{z_{c}} \frac{\partial W_{\Lambda}(Z)}{\partial Z} .
$$

We are led to the conclusion that $\varepsilon=1 / 2$ and $\eta=3 / 2$, which are precisely the values found in Euclidean $2 \mathrm{~d}$ gravity. Let us further remark that eq. (60) in this case becomes

$$
-\frac{\partial W_{\Lambda}(X)}{\partial \Lambda}=\text { const. } G_{\Lambda}\left(X, L_{2}=0\right),
$$

which differs from (44) by a derivative with respect to the cosmological constant. We will explain the reason for this difference below. Finally, eq. (61) becomes

$$
\int \mathrm{d} T \int \mathrm{d} Z G_{\Lambda}(X,-Z ; T) \frac{\partial W_{\Lambda}(Z)}{\partial Z}=\text { const. }
$$

which will be satisfied automatically if $\eta=3 / 2$ and $\varepsilon=1 / 2$, as we will show below.

We will now analyze a possible scaling limit of (50), assuming the canonical scaling $x=x_{c}(1-a X)$ and $g=g_{c}\left(1-\frac{1}{2} \Lambda a^{2}\right)$. In order that the equation have a scaling limit at all, $x_{c}, g_{c}$ and $w_{n s}\left(x_{c}, g_{c}\right)$ must satisfy two relations which can be determined straightforwardly from (50). The remaining continuum equation reads

$$
\begin{aligned}
a^{\varepsilon} \frac{\partial}{\partial T} G_{\Lambda}(X, Y ; T)= & -a \frac{\partial}{\partial X}\left[\left(X^{2}-\Lambda\right) G_{\Lambda}(X, Y ; T)\right] \\
& -a^{\eta-1} \frac{\partial}{\partial X}\left[W_{\Lambda}(X) G_{\Lambda}(X, Y ; T)\right] .
\end{aligned}
$$

The first term on the right-hand side of eq. (64) is precisely the one we have already encountered in our original model, while the second term is due to the creation of baby universes. Clearly the case $\eta<0$ (in fact $\eta \leq 1$ ) is 
inconsistent with the presence of the second term, i.e. the creation of baby universes. However, since $\eta<2$, the last term on the right-hand side of (64) will always dominate over the first term. Once we allow for the creation of baby universes, this process will completely dominate the continuum limit. In addition we get $\varepsilon=\eta-1$, in agreement with (61). It follows that $\eta>1$ and we conclude that $\varepsilon=1 / 2, \eta=3 / 2$ are the only possible scaling exponents if we allow for the creation of baby universes. These are precisely the scaling exponents obtained from two-dimensional Euclidean gravity in terms of dynamical triangulations, as we have already remarked. The topology changes of space have induced an anomalous dimension for $T$. If the second term on the right-hand side of (64) had been absent, this would have led to $\varepsilon=1$, and the time $T$ scaling in the same way as the spatial length $L$.

In summary, in the case $(\eta, \varepsilon)=(3 / 2,1 / 2)$ eq. (64) leads to the continuum equation

$$
\frac{\partial}{\partial T} G_{\Lambda}(X, Y ; T)=-\frac{\partial}{\partial X}\left[W_{\Lambda}(X) G_{\Lambda}(X, Y ; T)\right],
$$

which, combined with eq. (62), determines the continuum disc amplitude $W_{\Lambda}(X)$. Integrating (65) with respect to $T$ and using that $G_{\Lambda}\left(L_{1}, L_{2} ; T=\right.$ $0)=\delta\left(L_{1}-L_{2}\right)$, i.e.

$$
G_{\Lambda}\left(X, L_{2}=0 ; T=0\right)=1,
$$

we obtain

$$
-1=\frac{\partial}{\partial X}\left[W_{\Lambda}(X) \frac{\partial}{\partial \Lambda} W_{\Lambda}(X)\right]
$$

Since $W_{\Lambda}(X)$ has length dimension $-3 / 2$, i.e. $W_{\Lambda}^{2}(X)=X^{3} F(\sqrt{\Lambda} / X)$, the general solution must be of the form

$$
W_{\Lambda}(X)=\sqrt{-2 \Lambda X+b^{2} X^{3}+c^{2} \Lambda^{3 / 2}} .
$$

From the very origin of $W_{\Lambda}(X)$ as the Laplace transform of a disc amplitude $W_{\Lambda}(L)$ which is bounded, it follows that $W_{\Lambda}(X)$ has no singularities or cuts for $\operatorname{Re} X>0$. This requirement fixes the constants $b, c$ in (68) such that

$$
W_{\Lambda}(X)=b\left(X-\frac{\sqrt{2}}{b \sqrt{3}} \sqrt{\Lambda}\right) \sqrt{X+\frac{2 \sqrt{2}}{b \sqrt{3}} \sqrt{\Lambda}},
$$

where the constant $b$ is determined by the model-dependent constant in (62). This expression for the disc amplitude agrees after a rescaling of the cosmological constant with the amplitude $W_{\Lambda}^{(e u)}(X)$ from 2 d Euclidean quantum gravity,

$$
W_{\Lambda}(X)=\left(X-\frac{1}{2} \sqrt{\Lambda}\right) \sqrt{X+\sqrt{\Lambda}}
$$


With $W_{\Lambda}(X)$ substituted into (65), the resulting equation is familiar from the usual theory of $2 \mathrm{~d}$ Euclidean quantum gravity, where it has been derived in various ways $[18,35,36]$, with $T$ playing the role of geodesic distance between the initial and final loop.

Before showing that the anomalous scaling of the proper time - once baby universes are allowed - leads to an intrinsic fractal space-time dimension of four (rather than two), let us comment on the difference between the equations for the amplitudes (59)-(61) for $(\eta, \varepsilon)=(-1,1)$ and $(\eta, \varepsilon)=(3 / 2,1 / 2)$ respectively. In the first case there are no baby universes and eq. (59) entails that only macroscopic loops at a distance $T$ from the entrance loop are important (as illustrated in Fig. 3). On the other hand, the term $\partial W_{\Lambda}(Z) / \partial Z$ which describes the presence of these macroscopic loops is absent in eq. (60). This is consistent with eq. (62), which shows explicitly that the length of the upper loop in Fig. 3 remains at the cutoff scale, and therefore can never become macroscopic. It is also consistent with the abundance of baby universes, since at any point in space-time the probability for creating a little "tip" of cut-off size will dominate. At the same time, the right-hand side of eq. (59), that is, eq. (63), will play no

role when $1<\eta<2$, being simply equal to a constant. This latter property is satisfied automatically, as can be seen by using an equation analogous to (65) for the exit instead of the entrance loop. Thus eq. (63) becomes proportional to

$$
\int_{0}^{\infty} \mathrm{d} T \frac{\partial}{\partial T} G\left(X, L_{2}=0 ; T\right)=\text { const. }
$$

proving our previous assertion.

\subsection{THE FRACTAL DIMENSION OF EUCLIDEAN 2D GRAVITY}

If we allow for baby universe creation, the fractal structure of space-time is determined by (65) and (70), where $T$ is the time separating the entrance and exit loops. As already mentioned, these are exactly the equations governing Euclidean quantum gravity, if one replaces $T$ by the geodesic distance between the two loops.

One can solve eq. (65) in the same way as its Lorentzian analogue (36) was solved by (37)-(39). We have

$$
G_{\Lambda}(X, Y ; T)=\frac{W_{\Lambda}(\bar{X}(T ; X))}{W_{\Lambda}(X)} \frac{1}{\bar{X}(T ; X)+Y},
$$

where $\bar{X}(T ; X)$ is the solution to the characteristic equation

$$
\frac{d \bar{X}}{d T}=-W_{\Lambda}(\bar{X}), \quad \bar{X}(T=0)=X .
$$


This equation can be solved in terms of elementary functions. In particular, one finds

$$
\bar{X}(T ; X=\infty) \propto \sqrt{\Lambda} \operatorname{coth}^{2}\left(\alpha \Lambda^{1 / 4} T\right), \quad \alpha=\frac{1}{2} \sqrt{3 / 2} .
$$

We may now define a two-point function by contracting the entrance loop to a point and closing the exit loop by the disc amplitude. This is shown in Fig. 3, except that now the entrance loop must be contracted 1 . The two-point function $G_{\Lambda}(T)$ in Euclidean gravity can be interpreted as the (unnormalized) average over all geometries with two marked points which are separated by a geodesic distance $T$. At the discretized level it is defined by an equation analogous to (55), but without summing over $t$,

$$
G(t ; g)=\sum_{l} G\left(l_{1}, l ; g ; t\right) l w(l, g) .
$$

As in eq. (58), the continuum limit will be dominated by the non-scaling part of $w(l ; g)$, i.e. by small $l$, and the continuum two-point function is simply given by

$$
G_{\Lambda}(T) \sim G_{\Lambda}\left(L_{1}=0, L_{2}=0 ; T\right) .
$$

Using (72) and the Laplace transforms of $G_{\Lambda}$ and $W_{\Lambda}$ we obtain

$$
G_{\Lambda}(T)=\oint \frac{\mathrm{d} X}{2 \pi i} \oint \frac{\mathrm{d} Y}{2 \pi i} \frac{W_{\Lambda}(\bar{X}(T ; X))}{W_{\Lambda}(X)} \frac{1}{\bar{X}(T ; X)+Y} .
$$

With the help of the characteristic equation (73) this leads to

$$
G_{\Lambda}(T) \sim \frac{\mathrm{d}}{\mathrm{d} T} \oint \frac{\mathrm{d} X}{2 \pi i} \frac{\bar{X}(T ; X)}{W_{\Lambda}(X)} .
$$

The contour of integration can be deformed to infinity and we obtain from (74) that

$$
G_{\Lambda}(T) \sim \Lambda^{3 / 4} \frac{\cosh \alpha \Lambda^{1 / 4} T}{\sinh ^{3} \alpha \Lambda^{1 / 4} T} .
$$

This two-point function may be viewed as the partition function for universes with two marked points separated by a geodesic distance $T$. Since

\footnotetext{
${ }^{4}$ One cannot a priori contract both loops, since the two points in the two-point function are separated by a geodesic distance $T$, and there may be many points at distance $T$ from the entrance point, as shown in Fig. 3 . They will in general form several connected loops. However, after solving the model, it turns out that one can just contract the exit loop and obtain the two-point function. The reason is that $w(x, g)$ contains a non-universal part, which again implies that a typical disc amplitude in Fig. 3 will be of cut-off size. This is precisely the contents of eq. (60), whose non-scaling part is given by $\partial w_{n s} /\left.\partial z\right|_{z=x_{c}}$.
} 
we wanted to solve Euclidean gravity, this is our final result. Given (79), we can calculate the average space-time volume of such a universe,

$$
\langle V\rangle_{T}=T^{4} F\left(\Lambda^{1 / 4} T\right), \quad F(0)>0 .
$$

The function $F$ is again expressible in terms of elementary functions and one finds

$$
\langle V\rangle_{T} \approx T^{4} \quad \text { for } \quad T<1 / \Lambda^{1 / 4} .
$$

This formula shows that the intrinsic fractal dimension of $2 \mathrm{~d}$ Euclidean quantum space-time is four, whereas a similar derivation in the case of Lorentzian gravity yields [11]

$$
\langle V\rangle_{T} \approx T^{2} \quad \text { for } \quad T<1 / \Lambda^{1 / 2} .
$$

The discrepancy in dimension can be explained purely in terms of the baby universe structure: since at each point of the two-dimensional Lorentzian surface a baby universe can branch off, the resulting fractal Euclidean spacetime has twice the intrinsic dimension.

\section{Euclidean quantum gravity}

\subsection{SOME GENERALITIES}

In the last section we arrived at the $2 \mathrm{~d}$ Euclidean gravity theory through an "extension" of the Lorentzian model. Euclidean quantum gravity can of course be defined independently, as the quantization of classical gravity on the space of all Riemannian metrics (of positive definite signature) instead of the space of (indefinite-signature) Lorentzian metrics. In two dimensions, Euclidean gravity has a well-defined continuum path-integral formulation. Choosing a conformal gauge-fixing leads to the so-called Liouville gravity. Certain aspects of this theory can be solved by a bootstrap approach. In higher dimensions the path-integral approach to Euclidean quantum gravity is problematic, since the Einstein-Hilbert action is unbounded from below. There are various ways of tackling this problem: analytically continuing the unstable modes, adding stabilizing higher-derivative terms to the action, or defining the theory non-perturbatively via a lattice regularization, such that the action is bounded for any finite lattice volume.

One example of the latter is the dynamical-triangulations approach, which has the added bonus of being exactly soluble by combinatorial methods in two dimensions. Its continuum limit agrees with continuum Liouville quantum gravity, wherever the two formulations can be compared. We are in fact in the unusual situation that the lattice approach can address and answer more questions than the continuum methods. We will not review 
the combinatorial approach here since the results (for pure gravity) were already obtained in Section 3, starting from Lorentzian gravity. For a detailed description we refer to [8], chapter 4. The generalization of this Euclidean lattice path integral to higher dimensions is straightforward and shares two virtues with the $2 \mathrm{~d}$ case: calculating the partition function for gravity is again turned into a combinatorial problem, and the model is well-suited for numerical simulations. We have by now a good qualitative understanding of the phase structure of Euclidean dynamically-triangulated gravity in $d=3,4$, although complete analytical solutions of the discretized models are still missing. However, the combinatorial nature of the partition function gives us some hope that progress can be made also in these cases.

\subsection{DYNAMICAL TRIANGULATIONS}

This lattice approach shares many elements with lattice regularizations of ordinary quantum field theory. The main difference lies in the fact that the geometric degrees of freedom become dynamical and the lattices are therefore no longer part of the inert background structure. The geometric quantum fluctuations must be taken properly into account when building discretized models of matter interacting with quantum gravity. The fieldtheoretical, non-perturbative Euclidean path integral of such a theory takes the general form

$$
Z\left[G, \Lambda,\left\{\beta_{i}\right\}\right]=\int \mathcal{D}\left[g_{\mu \nu}\right] \mathcal{D} \phi_{i} e^{-S_{\text {matter }}\left[g_{\mu \nu}, \phi_{i} ;\left\{\beta_{i}\right\}\right]-S_{E H}\left[g_{\mu \nu} ; G, \Lambda\right]},
$$

where the integration is over equivalence classes of metrics $\left[g_{\mu \nu}\right]$ and matter fields $\phi_{i}$. The action contains a matter part $S_{\text {matter }}$, depending on a set of matter couplings $\left\{\beta_{i}\right\}$, and a purely geometric part, given by the EinsteinHilbert action with a cosmological term,

$$
S_{E H}\left[g_{\mu \nu} ; G, \Lambda\right]=\frac{1}{16 \pi G} \int d^{d} x \sqrt{\operatorname{det} g}(2 \Lambda-R) .
$$

In (83) we have omitted possible boundary terms. Except in $d=2$, expressions of the kind (83) have remained formal, due to the absence of a suitable diffeomorphism-invariant integration measure. The lattice formulation is an attempt to remedy this situation, by using an intermediate regularization of the non-perturbative path integral (83) (see [37, 8] for reviews). Defining a discrete regularization consists of several steps:

- a discretization of the individual metric manifolds, together with a definition of discretized geometric "observables", such as lengths, volumes and (scalar) curvature. These are necessary for obtaining a discretized version of the action, and for analyzing the physical properties of the theory in terms of scaling relations and correlation functions. 
- a suitable choice of an integration measure on the space of discretized geometries (i.e. equivalence classes of metrics), such that the discrete path integral converges.

- a discretization of the matter sector, which will be closely related to standard lattice-regularizations in field theory.

Let us now describe the dynamical-triangulations regularization of Euclidean quantum gravity. It consists in replacing the $d$-dimensional Riemannian metric continuum manifold by a simplicial manifold constructed from equilateral $d$-dimensional simplices of (geodesic) edge length $a$. (A simplex is a point in $d=0$, an edge in $d=1$, a triangle in $d=2$, a tetrahedron in $d=3$, etc.) Using Regge's prescription [38], all quantities can be expressed as functions of the squared edge lengths. For example, the curvature depends on local deficit angles, which in turn are expressible in terms of edge lengths. A simplicial complex is obtained by gluing together $d$-simplices pairwise along $(d-1)$-dimensional faces (which are themselves $(d-1)$-simplices). Since, unlike in Regge calculus, our edge lengths are not variable, all $d$-simplices have the same size, and the total volume of the simplicial complex is simply proportional to the number $N_{d}$ of such cells. Each $d$-simplex is built from simplices of lower dimensionality. It contains $d+10$ simplices (vertices), $(d+1) d / 21$-simplices (links) etc. A lower-dimensional subsimplex is in general shared by a number of $d$-simplices, called the order of the subsimplex. A simplicial complex is a simplicial manifold if the neighbourhood of any $p$-simplex $(p<d)$ has the topology of a $(d-p-1)$ dimensional sphere. Physically the manifold requirement may be viewed as a regularity condition at the cut-off scale, which will be convenient to use in our construction. The numbers $N_{k}$ of (sub-)simplices of dimension $k \leq d$ are not independent, but (due to the regularity requirement) must satisfy a set of so-called Dehn-Sommerville relations, namely,

$$
N_{i}=\sum_{k=1}^{d}(-1)^{k+d}\left(\begin{array}{c}
k+1 \\
i+1
\end{array}\right) N_{k},
$$

together with the Euler constraint

$$
\sum_{k=0}^{d}(-1)^{k} N_{k}=\chi
$$

For fixed Euler number $\chi$ and $d=2$, all $N_{k}$ can be expressed as functions of the single variable $N_{2}$, say. For $d=3,4$, two of the $N_{k}$ are independent.

A triangulation $T$ together with an assignment of geodesic edge lengths and flat simplex interiors may be viewed as a piecewise linear manifold, and provides an explicit coordinate-independent representation of a metric 
manifold. In this way each triangulation corresponds to a unique equivalence class of metrics (albeit of piecewise-linear, and not of differentiable type).

When it comes to numerical simulations, it is often convenient to assign a label to each vertex. From the list of vertex labels for all $d$-simplices the whole manifold can be reconstructed. Since the labels themselves have no physical meaning, the labelling introduces a redundancy. Invariance under permutations of the labels may loosely be regarded as a discrete analogue of the diffeomorphism invariance of a differentiable manifold. Also the lowerdimensional subsimplices are characterized by their vertex labels. Moreover, the regularity requirement implies that we cannot have two different (sub-)simplices with the same set of vertex labels. For each triangulation $T$ with $N_{0}$ vertices the number of different labellings equals $N_{0} ! / C(T)$, where $C(T)$ is the order of the automorphism group of $T$. We can therefore distinguish between labelled triangulations $\tilde{T}$ and abstract unlabelled triangulations $T$. As mentioned above, different T's (with fixed topology) correspond to different equivalence classes of piecewise linear metrics assigned to the underlying manifold, and allow us to work directly with a reparameterization-invariant set of geometries.

Since the simplices are flat on the inside, curvature is located (distributionally) at simplices of lower dimension. Circulating around a $(d-2)$ dimensional simplex, the contributing angles will in general not add up to $2 \pi$. The resulting deficit angle depends on the number of simplices meeting at the subsimplex. We conclude that the curvature is concentrated at the $(d-2)$-dimensional subsimplices of the triangulation. The Einstein-Hilbert action for a dynamically triangulated manifold in dimension $d$ assumes the simple form

$$
S_{E H}=\kappa_{d} N_{d}-\kappa_{d-2} N_{d-2},
$$

with the two dimensionless coupling constants $\kappa_{d}$ and $\kappa_{d-2}$. As usual in lattice regularizations, the lattice spacing $a$ has disappeared from the formulation and will have to be reintroduced in the scaling limit. We are using the Einstein-Hilbert action because of its simplicity; one could in principle consider also the inclusion of higher-order curvature terms.

With each simplicial lattice described above one can associate a dual lattice, whose vertices are located at the centres of the simplices of the original lattice. In a similar way we can associate to each $p$-simplex a dual object of dimension $d-p$. For example, the dual links connect the centres of neighbouring simplices, and the dual of a $(d-2)$-simplex is a closed loop (a two-dimensional object) whose length is equal to the number of $d$-simplices of the original lattice which share the $(d-2)$-simplex. Since in $d$ dimensions each simplex has $d+1$ neighbours, the dual lattices have the form of graphs 
of a scalar $\phi^{\mathbf{d}+\mathbf{1}}$-theory (that is, all their vertices are $(d+1)$-valent), but with a local $d$-dimensional topological structure.

The simplicial structures described above possess a natural notion of length for any path connecting two points, since the equivalence class of metrics is uniquely determined. To simplify matters, we will only consider certain sets of discretized paths on the simplicial manifold. The first set is given by paths which run along the links of the original simplicial lattice, and the other by paths running along the links of the dual lattice. In either case we may define a distance between points on the lattice or its dual as the number of edges of the shortest path connecting the two. At first glance these definitions seem different from the standard notion of a geodesic distance, but they all coincide in the scaling limit, up to trivial numerical factors 5 . Numerical tests of this assumption will be discussed below.

\subsection{THE FUNCTIONAL INTEGRAL}

The association of triangulations with equivalence classes of metrics motivates the use of the discrete sum over $d$-dimensional triangulations as a discretized analogue of the diffeomorphism-invariant integration measure in (83),

$$
\int \mathcal{D}\left[g_{\mu \nu}\right] \rightarrow \sum_{T} \frac{1}{C(T)}
$$

where the sum is taken over unlabelled simplicial manifolds. The need for including the symmetry factor $C(T)$ becomes apparent when one rewrites the right-hand side of (88) as a sum over labelled triangulations $\tilde{T}$,

$$
\sum_{T} \frac{1}{C(T)} \rightarrow \sum_{\tilde{T}} \frac{1}{N_{0}(\tilde{T}) !}
$$

In order that a discretized path integral with this choice of measure lead to a theory with a well-defined thermodynamic limit, the number of triangulations with fixed volume $N_{d}$ should grow at most exponentially with $N_{d}$ as $N_{d} \rightarrow \infty$. This is not the case unless we fix the space-time topology (usually to that of a sphere $\mathrm{S}^{d}$ ); otherwise the growth is factorial. This property has been proven for $d=2$ and arbitrary topology [39] and for simply connected manifolds in $d=3,4[24,25]$.

\footnotetext{
${ }^{5}$ The situation is the same as for a regular $2 \mathrm{~d}$ quadratic lattice in flat space: if we are only allowed to connect vertices along the lattice links, the lattice distance between different lattice points can differ by as much as a factor $\sqrt{2}$ from the Euclidean distance in flat space. However, in the scaling limit the rotational symmetry of the original field theory will be restored on the lattice and the two notions of distance will only differ by an overall factor.
} 
It is worthwhile pointing out that the simple choices (87) for the action and (88) for the measure lead to a partition function of the form

$$
Z\left(g_{d}, g_{d-2}\right)=\sum_{T} \frac{1}{C(T)} g_{d}^{N_{d}(T)} g_{d-2}^{N_{d-2}(T)},
$$

where $g_{d}=-\log \kappa_{d}$ and $g_{d-2}=\log \kappa_{d-2}$. Eq. (90) shows that the partition function is the generating function for the number of triangulations (of fixed topology) with given numbers $N_{d}$ and $N_{d-2}$ of $d$ - and ( $d-2$ )-simplices. We thus reach the surprising conclusion that even in dimension $d>2$, quantum gravity can be formulated as a (relatively simple) counting problem.

\subsection{INCLUSION OF MATTER FIELDS}

The discretization of matter fields coupled to dynamical triangulations is achieved by standard lattice field-theoretical methods. The simplest types of matter fields one can study are either scalar fields or (Potts) spin fields, carrying a discrete space-time label. One may also combine several fields of this type. The matter fields can be located at the vertices of the triangulation or at the centres of the $d$-simplices (that is, at the dual vertices). The interactions are typically of the form of nearest-neighbour interactions, where the "nearest neighbours" are the vertices that are one link length (or one dual link length) away from the original vertex. We expect these two formulations to become equivalent in the scaling limit. Some typical examples of matter actions are

$$
S_{I}=\frac{\beta}{2} \sum_{\{i j\}}\left(\delta_{\sigma_{i} \sigma_{j}}-1\right),
$$

where the $\sigma_{i}$ are a set of Potts spins, or the Gaussian action

$$
S_{g}=\sum_{\{i j\}}\left(\phi_{i}-\phi_{j}\right)^{2}
$$

for a massless scalar field $\phi$. Note that we did not need to include a coupling constant in front of the action (92). The massless scalar field can always be rescaled by a factor, which can then be absorbed by a redefinition of the coupling constants of the geometric sector. The coupling of Ising spins (Potts spins $\sigma_{i}$ with $i=1,2$ ) and Gaussian fields to the 2d Lorentzian gravity model proceeds in a manner identical to (91) and (92).

In higher dimensions we will also consider the coupling to gauge fields. As usual these are associated with the one-dimensional edges of the triangulation, and there are again two alternative formulations, depending on

whether the links or the dual links are used. The gauge field action is more 
complicated and we postpone its discussion to Section 6. The inclusion of fermionic degrees of freedom on a random manifold remains an open problem, particularly in higher dimensions. It requires the definition of a spin connection on a simplicial manifold (c.f. [40]). The problem was solved in $d=2$ [41]. In this case one can prove that a system of Wilson fermions on a triangulated manifold can be "bosonized" and represented as a system of Ising spins on the manifold $[42,41]$.

The discretized path-integral measure contains also a sum over matter fields. In the case of spin variables, the sum is simply taken over all possible spin configurations. For continuous fields like the scalar fields above, one may consider non-trivial integration measures which introduce an additional coupling to geometry. This possibility does not exist in field theories on fixed, regular lattices, where such a dependence is always trivial. It leads to some subtleties in the case of gauge fields, as we will discuss later.

The simplest and most extensively studied example of a dynamically triangulated theory is that of Euclidean gravity on a two-sphere. The fundamental building blocks in this case are equilateral triangles (2-simplices). Triangles are glued together pairwise along edges (1-simplices), and each triangle has exactly three neighbours. The dual lattice is thus equivalent to a planar $\phi^{\mathbf{3}}$-diagram. The curvature is localized at the vertices (0-simplices) which in general are shared by many triangles, each contributing $\pi / 3$ to the total angle around the vertex. The regularity requirement introduced above prohibits configurations where a vertex is its own neighbour or where two vertices are connected by more than one link (in other words, closed loops of link length one or two are forbidden).

The Lorentzian gravity model introduced in Section 2.1 may be viewed as a restricted version of the dynamically triangulated Euclidean model, since the triangulations contributing to the Lorentzian state sum constitute a subset of those appearing in the Euclidean system defined above. Recall also our construction of Euclidean from Lorentzian gravity in Section 3.1, by allowing for additional baby-universe branching. Again the set of all such geometries is a subset of all $2 \mathrm{~d}$ simplicial manifolds, but both continuum theories coincide. What is at work in this latter case is "universality", which ensures that the continuum limit is to a large extent independent of the short-distance details characterizing the class of triangulations we sum over. The universality properties of Euclidean $2 \mathrm{~d}$ quantum gravity are well studied. For example, one may relax the manifold regularity condition to obtain a much larger class of simplicial complexes, whose continuum limit is still Euclidean quantum gravity. Only drastic modifications, like the suppression of baby universes, can move the system to a different universality class with a different critical behaviour and therefore a different continuum limit. Also around the fixed point leading to Lorentzian $2 \mathrm{~d}$ gravity one 
finds an independence of short-distance details. Universality with respect to a change of fundamental building blocks and the inclusion of higher curvature has been demonstrated in [28]. It is also encountered in a recently developped procedure for obtaining Lorentzian from Euclidean quantum gravity by removing baby universes [17]. There one ends up with a generalized class of triangulations (compared to the original Lorentzian model), but the continuum limit is still the same.

\section{Numerical setup}

\subsection{MONTE CARLO METHOD AND ERGODIC MOVES}

Even in two dimensions, there are a number of issues of the matter-coupled theory that presently can only be addressed by numerical methods. Lorentzian gravity coupled to Ising spins has not yet been solved analytically. In matter-coupled Euclidean 2d quantum gravity, analytical considerations have not yet led to a determination of the fractal dimension of space-time (there are various suggestions leading to different answers), nor do we know what happens beyond the infamous $c=1$ barrier, where analytical calculations break down. In higher dimensions, we do not even have analytical solutions of the pure-gravity models. In all of these situations, numerical simulations of the systems come in handy. They can answer specific questions and lead to unexpected results which in turn can inspire further analytical work.

Numerical simulations of simplicial gravity have been the subject of a number of reviews (see [9, 43] for annual updates and [44] for more information on the computer codes used in the programs). Here we will only sketch the methods and use the simplest case of $2 \mathrm{~d}$ Euclidean gravity as an illustration. Most of what we will have to say carries over to $2 \mathrm{~d}$ Lorentzian gravity with only minor modifications.

As explained in the previous section, the discretized theory can be described by the partition function

$$
Z=\sum_{\tilde{T}} \frac{1}{N_{0}(\tilde{T}) !} e^{-S_{E H}(\tilde{T})} \sum_{\phi_{i}} e^{-S_{\text {matter }}\left(\phi_{i}\right)},
$$

where $S_{E H}$ is the discretized Einstein-Hilbert action (87), and the first sum is taken over all labelled triangulations of fixed spherical topology. For $d=2$, the geometric part of the action simplifies and takes the form

$$
S_{E H}=\mu N_{2},
$$

up to an additive constant proportional to the Euler number $\chi$, with $\mu=$ $\left(\kappa_{2}-\kappa_{0}\right) / 2$. In numerical simulations, it is simpler to use the labelled instead of the unlabelled triangulations. The partition function (93) is the 
analogue of the grand canonical partition function in statistical mechanics. The cosmological constant $\kappa_{d}$ plays the role of a thermodynamic potential for the number of simplices. For general $d$, we may rewrite (93) as

$$
Z=\sum_{N_{d}} e^{-\kappa_{d} N_{d}} Z_{N_{d}}
$$

where $Z_{N_{d}}$ can be interpreted as the partition function at fixed volume. A gravity-matter configuration $C$ is uniquely specified by a geometry in the form of a labelled triangulation and by the values of all matter field variables. Each configuration enters the statistical sum with a probability proportional to $e^{-S(C)}$, where $S(C)=S_{E H}+S_{\text {matter }}$. As usual in statistical mechanics, physical information can be obtained by measuring the averages of various operators in this ensemble. The fact that each configuration has a real positive weight makes it possible to study the system (93) by Monte Carlo methods. The goal is to construct a numerical "generator" which produces configurations $C$ with a probability $P(C) \sim e^{-S(C)}$.

Except for very few cases, where a direct generation of all configurations is possible (e.g. for a conformal charge $c=-2$ in $2 \mathrm{~d}$ ), the standard way of obtaining such a generator is by means of a stochastic process (a Markov chain), which can be regarded as a random walk in configuration space. Since the random walk takes place on a computer, each step corresponds to the real time needed to perform such an operation. The stochastic process is defined by a function $W\left(C \rightarrow C^{\prime}\right) \geq 0$, giving the probability for a transition from a configuration $C$ to $C^{\prime}$ in one step. Assuming for simplicity that the configuration space is discrete, we have a normalization condition

$$
\sum_{C^{\prime}} W\left(C \rightarrow C^{\prime}\right)=1
$$

In most cases $W\left(C \rightarrow C^{\prime}\right)$ is chosen to vanish outside some "neighbourhood" of $C$. Starting from an initial state $p_{0}(C)$, the system after $n$ steps is characterized by a probability distribution $p_{n}(C)$, where

$$
p_{n+1}(C)=\sum_{C^{\prime}} p_{n}\left(C^{\prime}\right) W\left(C^{\prime} \rightarrow C\right) .
$$

The transition probability $W$ must satisfy two basic requirements,

1. ergodicity: any two configurations can be joined by a finite number of steps, and

2. detailed balance: the condition

$$
P(C) W\left(C \rightarrow C^{\prime}\right)=P\left(C^{\prime}\right) W\left(C^{\prime} \rightarrow C\right),
$$

relating $W\left(C \rightarrow C^{\prime}\right)$ to $W\left(C^{\prime} \rightarrow C\right)$. 
It follows from (98) that $W\left(C \rightarrow C^{\prime}\right)$ and $W\left(C^{\prime} \rightarrow C\right)$ are either both zero or both non-zero in which case they satisfy

$$
\frac{W\left(C \rightarrow C^{\prime}\right)}{W\left(C^{\prime} \rightarrow C\right)}=\frac{P\left(C^{\prime}\right)}{P(C)}
$$

These two requirements guarantee that the stochastic process has a unique asymptotic probability distribution $p_{\infty}(C) \sim P(C)$, which is the only eigenstate of the transition matrix $W$ with eigenvalue 1 ,

$$
\sum_{C^{\prime}} p_{\infty}\left(C^{\prime}\right) W\left(C^{\prime} \rightarrow C\right)=p_{\infty}(C)
$$

All other eigenvalues are strictly smaller than 1 . This implies that - independent of the initial configuration - the system will reach the asymptotic distribution after infinitely many steps. Note that the asymptotic distribution has the desired probability distribution. The rate at which the system approaches this limiting distribution depends on the other eigenvalues of $W$. The contributions from other eigenstates decay exponentially with the number of steps. The second-largest eigenvalue $\lambda_{1}$ provides us with an estimate of the autocorrelation time $T=-1 / \log \lambda_{1}$. When the number $n$ of steps is $\gg T$, we can assume that the distribution is asymptotic. Typically the autocorrelation time behaves like $T \sim f^{\delta}$, where $f$ counts the number of degrees of freedom of the system and $\delta$ is a dynamical exponent, depending on the details of the algorithm.

In a practical implementation the system starts from some configuration $C_{0}$. During the first step, it changes to $C_{1}$ with probability $W\left(C_{0} \rightarrow C_{1}\right)$, or remains at $C_{0}$ if the change is not accepted. After $n \gg T$ steps it reaches a configuration $C_{n}=C^{(1)}$ with a probability proportional to $P\left(C^{(1)}\right)$. This configuration is the starting point of a new process, during which another sufficiently large number of steps is performed and a new configuration $C^{(2)}$ generated. Repeating this process we create a (finite) set of configurations $\left\{C^{(1)}, \ldots, C^{(N)}\right\}$, where $N$ depends on the computer time we spend on the project. The average of any operator in the ensemble of configurations defined by the probability distribution $P(C)$ is approximated by an average over this finite sample of "typical" configurations.

The requirements listed above by no means define the stochastic process uniquely. We are interested in efficient algorithms which minimize $\delta$, and can produce a large number of independent configurations in the shortest possible time. There is no simple way to guess at the outset whether an algorithm is efficient or not. Each problem must be treated individually and the autocorrelation time measured numerically. There are some general guidelines which one usually follows when creating a new algorithm. As discussed above, the efficiency depends on the choice of the transition 
probabilities $W\left(C \rightarrow C^{\prime}\right)$. We would like the algorithm to have a high "mobility", that is, a high probability that a configuration of the system will change at each step. This means that the set $\left\{C^{\prime}\right\}$ of configurations which can be reached from a given $C$ should be limited. If it is too large, each transition probability will be small, implying that the configuration will most likely not change. On the other hand, the set $\left\{C^{\prime}\right\}$ must be large enough to ensure ergodicity.

The detailed-balance condition (99) implies that if both transitions are to be reasonably probable, we must choose the set $\left\{C^{\prime}\right\}$ such that its elements have similar probabilities to that of $C$ (equivalently, have a small action difference $\left.\left|S(C)-S\left(C^{\prime}\right)\right|\right)$. On a fixed lattice, small differences in the action are usually realized by considering at each step only local changes in the field variables, for instance, changing only one variable, while keeping all the others fixed. When a more complicated change is attempted, the action difference is in general large and proportional to the volume of the system. Local changes are not very efficient when the typical fluctuations are long-ranged, as happens close to a continuous phase transition. Creating a Monte Carlo algorithm which is at the same time ergodic and has a reasonably small autocorrelation time even in the critical region is an art.

The first step in constructing an algorithm for simplicial gravity is to define a method of coding the configurations. From a numerical point of view it is natural to work with labelled rather than unlabelled triangulations, because otherwise it is almost impossible to keep track of the (dynamical) connectivity. To code the geometric structure of such a configuration it is in principle sufficient to have a list of the vertex labels of all simplices (of all dimensions) of the triangulation. Two vertices are neighbours if they belong to the same simplex. From this list we can reconstruct the complete geometry of the system. The fact that we have a list of simplices means in practice that we have also assigned labels to the simplices. Two simplices are neighbours if they share a $(d-1)$-simplex or, equivalently, $d$ vertices. In addition, we can make a list of all subsimplices and count their order. The process of reconstruction may still be complicated and it is often useful to keep at each step even more information, in the extreme case the lists of all subsimplices. The more information we keep, the easier it is to reconstruct local properties of the geometry. However, it also means that during geometry updates more data will have to be changed.

The next step consists in constructing the stochastic process described above. In our case this amounts to defining a set of "moves", which connect configurations with different geometry and matter content. Usually these two changes are performed separately: changes of the matter fields are performed for a fixed geometric configuration, by techniques very similar to those used for fixed lattices. For spin systems there exist highly effective 
cluster algorithms [45] with very short autocorrelation times. Cluster algorithms are special in that they permit global changes of the spin configurations. For Gaussian fields, no such algorithms exist and the field variables are usually updated sequentially. (There are some methods to shorten their autocorrelation time.) Generally speaking, the autocorrelation times for Gaussian fields are much longer than those for spin systems. It is therefore necessary to repeat the updating of all fields on the manifold several times in order to obtain independent field configurations.

In addition, we must define a set of geometric moves which generate local changes of the geometry, while preserving its underlying topology. Such moves are necessary even in the absence of matter. "Local changes" means that only a small region of the manifold is affected at each step. Again, it is important that the set of moves be ergodic in the space of all possible triangulations. For simplicial manifolds, one possible choice is given by the so-called Alexander moves [46]. In practical applications most algorithms for a $d$-dimensional geometry are based on a finite subset of moves containing $d+1$ operations. We will describe this set of operations in the case of $2 \mathrm{~d}$ and postpone its generalization to higher $d$ to Section 6 .

The first operation is called a flip and involves two neighbouring triangles. The triangles are denoted by their vertex labels, $\{123\}$ and $\{124\}$, and share the link $\{12\}$. It is important that the four labels are all distinct (excluding $\{3\}=\{4\}$, say) and that the vertices $\{3\}$ and $\{4\}$ are not connected by a link. The flip consists in replacing this configuration by the two triangles $\{341\}$ and $\{342\}$. In other words, the link $\{12\}$ is "flipped" to the link $\{34\}$. The restrictions imposed above guarantee that this move does not produce a pathological triangulation. The inverse of the flip operation is again a flip. Note that the flip move takes place entirely inside the link loop $\{1324\}$, whose boundary it leaves unchanged. Since two simplices are replaced by two others, the flip is sometimes called a $(2,2)$-move. A flip can be performed almost everywhere in the manifold, giving rise to a different labelled manifold whose connectivity is changed locally. If matter fields are present at the vertices, one usually assumes that their values are unchanged. The transition weights $W\left(C \rightarrow C^{\prime}\right)$ and $W\left(C^{\prime} \rightarrow C\right)$ for a flip and its inverse can easily be computed from the detailed-balance condition. We will not be more specific here, since this depends on the details of the geometric coding (in particular, on how links are selected). A flip move leaves the numbers of triangles and vertices unchanged.

The second move adds a new vertex $\{4\}$ inside a triangle $\{123\}$. This move replaces the old triangle by the three triangles $\{124\},\{234\}$ and $\{314\}$, and leaves the manifold outside the "boundary" of $\{123\}$ unaffected. It is also known as a $(1,3)$-move. The new vertex is of order three, since it is shared by three triangles. The third move is the inverse of the $(1,3)$ - 

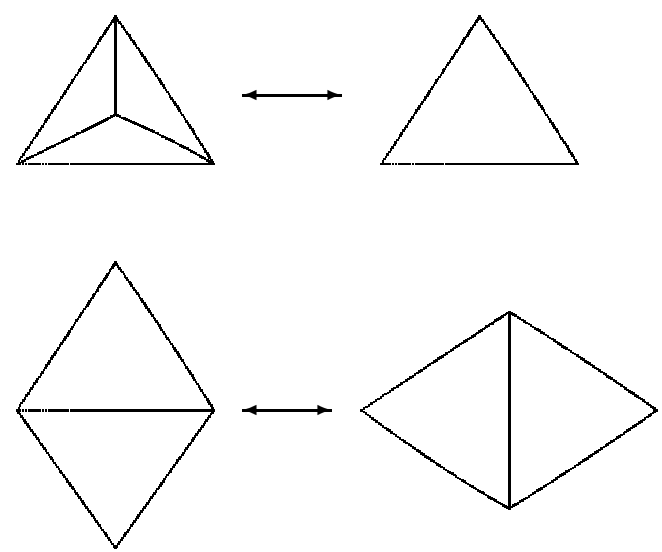

Figure 4. A set of three moves which is ergodic in the class of two-dimensional triangulations of fixed topology. The first diagram shows the $(3,1)$-move and its inverse. The second diagram shows the $(2,2)$ - or flip move. By itself, this move is ergodic in the class of triangulations of fixed volume $N_{2}$ and topology.

move. It is a $(3,1)$-move where a vertex of order three is removed, and the three triangles which share it are replaced by a single triangle. The $(1,3)$-move can be performed on each triangle of a manifold, but its inverse needs a vertex of coordination number three. It is obvious that in both cases the configurations before and after the move are two different labelled triangulations. If matter fields are present, a new field must be created at the new vertex generated during a $(1,3)$-move. This must be taken into account when the transition weights are calculated. The three types of moves are depicted in Fig. 1 .

The three operations just described leave the manifold topology unchanged. They form an ergodic set, which means that any two simplicial manifolds of the same topology can be related by a finite sequence of moves. In $2 \mathrm{~d}$ one can construct such a sequence explicitly. The set of ergodic moves is not unique, and alternative sets of local moves have been used in applications. For example, a point-splitting algorithm is described in [8]. The point-splitting move and its inverse (illustrated in Fig. 5) change the volume $N_{2}$ by \pm 2 . The $(1,3)$ - and $(3,1)$-moves are special cases and the (2,2)-move can be realized as a sequence of two point-splitting moves.

Our discussion so far suggests that we may set up a numerical simulation by generating configurations according to the probability distribution (93), and use the resulting sample of configurations to measure the quantities of interest. However, this is not really feasible, since the system described by (93) is an open system in the sense that arbitrarily large configurations may be produced by using one of the sets of geodesic moves described above. In practice, we must limit this size because of the obvious memory restrictions of a computer. A simple solution is to generate a set of configurations 

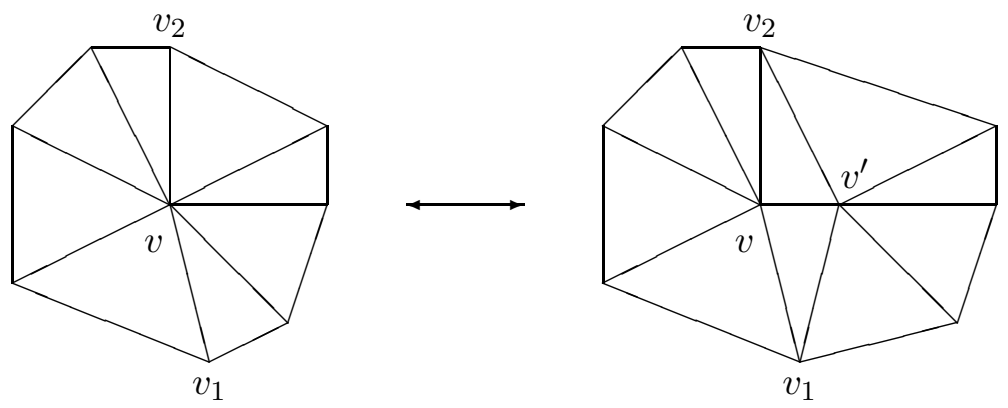

Figure 5. The point-splitting moves constitute an alternative set of ergodic moves.
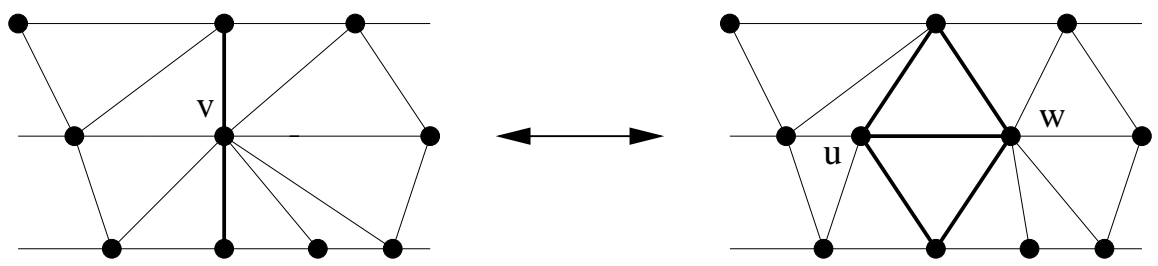

Figure 6. The move used in the Monte Carlo updating of a 2d Lorentzian geometry.

of fixed volume $N_{2}$, and repeat the experiment for various values of $N_{2}$. This is what one typically does in numerical simulations of field theory. For the case of Euclidean $2 \mathrm{~d}$ triangulations we are particularly lucky, since the $(2,2)$-flip move is already by itself ergodic in the space of all triangulations of fixed volume $N_{2}$, considerably simplifying the computer simulations. Unfortunately there is no similar result in higher dimensions; we will describe later the method used in this case. The flip move cannot be used in numerical simulations of the Lorentzian model described in Section 2.1, since it is not compatible with the causal structure. One uses instead a version of the point-splitting move (Fig. (6) to update the geometry (see [12] for details).

We conclude this section by describing another type of move which is associated with a large change in matter and geometry (while keeping $\Delta S$ small). It is motivated by the baby-universe structure of Euclidean simplicial gravity. Let us consider a two-dimensional triangulated manifold with the topology of a disc. It is characterized by its volume $n$ and boundary length $l$. For a random geometry these two numbers are not related since for any finite $l, n$ can become arbitrarily large. If we have two such discs, of volume $n$ and $N-n$, but with identical boundary lengths, we can identify 
their boundaries to create a closed simplicial complex of spherical topology and volume $N_{2}=N$. The smaller component is the baby universe and the larger one the parent universe. A typical spherical manifold observed in numerical simulations will contain many structures of this kind, of various sizes and lengths of boundaries, called "necks". The shortest possible neck has length three, and the associated baby universe will be called a minimal baby universe (minbu). We may also have baby universes characterized by longer (but finite and small) neck sizes.

The existence of baby universes opens a completely new possibility for updating the matter sector of the theory. Note that for a finite boundary length $l$, the interaction between the matter in the baby universe and the parent universe will be of order $l$. Take the example of Ising spins: even if we flip all spins inside the baby universe, the change in the action will only depend on the interactions across the boundary, and not the size $n$ of the baby universe. This is a completely different situation than on a regular lattice where large domains always have large boundaries. We may use this observation to define a matter update which induces a large change in magnetization and has a large acceptance. The efficiency of such an algorithm will depend on the typical baby universe size. One possible algorithm consists in searching the triangulation for a minimal neck and flip all spins inside the associated minbu with probability $W$. The same technique can be applied to massless scalar fields. Here we can perform two operations on the fields in the minbu: change all of their signs and/or add the same constant to all of them.

The existence of minbus can be exploited also in the geometric sector. For example, we may use the following three-step algorithm.

- Locate a minimal neck in the triangulation, and cut open the original spherical manifold along the neck. Both of the resulting discs are of the form of a triangulated sphere with a triangle removed.

- Close off both holes by a single triangle, to obtain two spherical triangulations of volumes $n+1$ and $N-n+1$ (if the original triangulation had volume $N$ ).

- Remove an arbitrary triangle from each of the two manifolds, and glue the resulting discs along their triangular boundaries.

We must compute the correct probability factors for such a move and eventually also include changes in the matter fields. Based on this idea, an extremely efficient "minbu surgery" algorithm has been constructed, shortening the autocorrelation times by three orders of magnitude [16]. The move does not even require a lot of geometric updating if it is accepted, since the connectivities of the manifold are changed only locally. The minbu surgery usually supplements the local moves described earlier, although in $2 \mathrm{~d}$ one may construct an algorithm that is based exclusively on the cutting 
and pasting of baby universes, if one permits also longer neck lengths. (A flip move may be viewed as a particular realization of this, if instead of minbus we consider baby universes with boundaries of length $l=4$.)

\subsection{OBSERVABLES IN 2D EUCLIDEAN GRAVITY}

The Monte Carlo method described above can be used to generate a set of uncorrelated configurations of volume $N_{2}$ which are distributed according to their Boltzmann weights $e^{-S}$. In order to understand the finite-size effects, one needs to perform the simulations at different values of $N_{2}$. The sizes that have been used in numerical simulations to date range from 1000 to 128000 simplices.

In $2 \mathrm{~d}$ it speeds up the calculations to extend the class of allowed triangulations to include also loops of link length one and two (corresponding to self-energy and tadpole subdiagrams on the dual lattice). Let us denote this extended class by $\mathcal{T}$ and the corresponding class of labelled triangulations by $\tilde{\mathcal{T}}$. Although the complexes constructed in this way are no longer simplicial manifolds, we can still keep the notions of global topology, of local neighbourhoods and of a geodesic distance. Likewise, the relations between the numbers $N_{k}$ remain unchanged. In some cases (including pure $2 \mathrm{~d}$ gravity), models based on this set of geometries can be compared directly with the analytic solutions of corresponding matrix models, where the exclusion of tadpole and self-energy subdiagrams corresponds merely to a finite renormalization of the bare coupling constants. On $\mathcal{T}$, the numerical simulations become even simpler. For fixed volume, the flip move is still ergodic and also the minbu surgery moves can be generalized. The advantage of using this class of triangulations is a reduction of the finite-size effects, since it turns out that the local restrictions on the connectivity do not affect the scaling properties of the system.

Our next step will be to describe the measurement of suitable "observables" on the ensemble of configurations generated by the Monte Carlo algorithm. The observables most easily obtained are the critical exponents related to the geometry or the matter fields. In two dimensions, one can sometimes obtain such observables analytically, and use them to test the validity of the numerical results. Recall that in $2 \mathrm{~d}$ we start from the partition function

$$
Z=\sum_{\tilde{\mathcal{I}}} \frac{1}{N_{0} !} \sum_{\phi_{i}} e^{-\mu V-S_{\text {matter }}\left(\phi_{i}\right)}=\sum_{V} e^{-\mu V} Z_{V},
$$

where $Z_{V}$ is the partition function at fixed volume. If the central charge of matter is $c<1$, it can be shown analytically that $Z_{V}$ behaves like

$$
Z_{V} \sim e^{\mu_{0} V} V^{\gamma_{s t r}-3}(1+\mathcal{O}(1 / V))
$$


for large $V$ and spherical topology. The subleading power contains the critical exponent $\gamma_{s t r}$, which has a known dependence on $c$ (see formula (125) below). The pure-gravity case corresponds to $\gamma_{s t r}=-1 / 2$.

There are other statistical systems whose partition function behaves like (102), most notably, various realizations of branched polymers [20, 47]. In these models, $\gamma_{s t r}$ is positive and $\leq 1 / 2$, and $V$ counts the number of vertices.

Measuring the distribution of minbu sizes for a triangulation of fixed volume $N_{2}=V$ is an efficient device for determining the critical exponent $\gamma_{s t r}[48,49]$. The average number $\langle b(n)\rangle_{V}$ of minbus with volume $0 \ll n<$ $V / 2$ is given by

$$
\langle b(n)\rangle_{V} \sim \frac{n Z_{n}(V-n) Z_{V-n}}{Z_{V}},
$$

since a minbu of size $n$ can be regarded as a spherical triangulation of volume $n+1$ with one marked triangle. (Since both $n$ and $V-n$ are assumed large we can neglect small corrections to the volume.) Using (102), we obtain

$$
\langle b(n)\rangle_{V} \sim V(n(V-n))^{\gamma_{s t r}-2} .
$$

Measuring $\gamma_{s t r}$ gives us information about the fractal structure of the theory and provides a simple test of algorithms in cases where its value is known. In all cases with $c<1$, one finds an excellent numerical agreement with the predicted values. From relation (104) we see that for $-1<\gamma_{s t r}<0$, the average minbu size $\langle n\rangle_{V}$ remains finite, but that $\left\langle n^{2}\right\rangle_{V} \sim V^{\gamma_{s t r}+1}$. This means that with growing $\gamma_{s t r}$ we will observe increasingly large fluctuations in minbu size. If $\gamma_{s t r}>0$ the average minbu size behaves like $\langle n\rangle_{V} \sim V^{\gamma_{s t r}}$.

Another important observable that can be studied by numerical simulations is the volume-volume correlator, which is a particular example of a geometric two-point function. In the continuum theory, it can be defined as

$$
G_{\Lambda}(R)=\int \mathcal{D}\left[g_{\mu \nu}\right] \mathcal{D} \phi e^{-S[g, \phi]} \iint \mathrm{d} \xi \mathrm{d} \xi^{\prime} \sqrt{g(\xi)} \sqrt{g\left(\xi^{\prime}\right)} \delta\left(d_{g}\left(\xi, \xi^{\prime}\right)-R\right),
$$

where $d_{g}\left(\xi, \xi^{\prime}\right)$ denotes the geodesic distance between two points $\xi$ and $\xi^{\prime}$, calculated with respect to the metric $g_{\mu \nu} . G_{\Lambda}(R)$ is the partition function for universes with two marked points separated by a geodesic distance $R$, which we already encountered in Section 3.2. In a discretized theory we can construct the analogous quantity

$$
G_{\mu}(r)=\sum_{\tilde{\mathcal{T}}} \sum_{\phi_{i}} e^{-\mu N_{2}-S_{\text {matter }}\left(\phi_{i}\right)} \sum_{i, j} \delta_{D(i, j), r},
$$

where $D(i, j)$ is now one of the integer-valued geodesic distances introduced at the end of Section 4.2. The two possibilities will in general differ by a 
finite scaling factor. For large $r$, one can show that $G_{\mu}(r)$ falls off exponentially like

$$
G_{\mu}(r) \sim e^{-m(\mu) r}, \quad r \gg 1 / m(\mu) .
$$

For small $r$ we expect

$$
G_{\mu}(r) \sim r^{1-\eta}
$$

where $\eta$ is the anomalous dimension of the two-point function. For $\mu \rightarrow \mu_{0}$ we make the ansatz $m(\mu) \sim\left(\mu-\mu_{0}\right)^{1 / d_{h}}$ where $d_{h}$ is another critical exponent of the theory. From the functional form of the partition function $G_{\mu}(r)$, one can derive the estimates $\langle V\rangle_{\mu} \sim 1 /\left(\mu-\mu_{0}\right)$ and $\langle r\rangle_{\mu} \sim 1 /\left(\mu-\mu_{0}\right)^{1 / d_{h}}$. From these one obtains a relation between the average linear extension and the average volume of the configuration, namely,

$$
\langle V\rangle_{\mu} \sim\langle r\rangle_{\mu}^{d_{h}}
$$

The exponent $d_{h}$ is called the cosmological Hausdorff dimension. It is a large-scale property of the average "quantum geometry" of the ensemble, and therefore need not coincide with the dimension of the individual triangulations.

The function $G_{\mu}(r)$ has been calculated analytically for pure gravity, as explained in Section 3.2, leading to $d_{h}=4$. As we have already pointed out, it is convenient in numerical simulations to work with triangulations of fixed total volume. The two-point functions at fixed and unrestricted volumes are related by a discrete Laplace transform. From definition (106) we have

$$
G_{\mu}(r)=\sum_{V} e^{-\mu V} G_{V}(r)
$$

where $G_{V}(r)$ is the partition function at fixed volume $V$. For a particular configuration of the system, $G_{V}(r)$ is measured as follows. Start with a (dual) vertex $i$ and find all (dual) vertices at (dual) distance 1 from it. By iterating this process, the triangulation is decomposed into shells characterized by their distance $r$ from the initial point $i$. Note that the shells will in general be disconnected. We can measure the total length of each shell, that is, the number of vertices in the shell. 6 This construction is repeated for all starting points and all configurations in the sample to obtain an averaged distribution. The resulting quantity $\langle n(r)\rangle_{V}$ is - up to a normalization a numerical estimate of $G_{V}(r)$. It is convenient to use the normalization condition

$$
\sum_{r} G_{V}(r)=V
$$

\footnotetext{
${ }^{6}$ Note that one could also measure the number of disconnected parts and/or the higher moments of their length distribution.
} 
which leads to an interpretation of $G_{V}(r)$ as the average volume of a shell with radius $r$ for a triangulation of volume $V$. For large $V$ one expects that

$$
G_{V}(r) \sim V^{1-1 / d_{h}} F(x),
$$

where $x=r / V^{1 / d_{h}}$ is the scaling variable. In terms of $x$, we have a normalization condition

$$
\int d x F(x)=1
$$

From an inverse Laplace transform of relation (107), we can deduce that for large $x, F(x)$ must behave like

$$
\log F(x) \sim x^{\frac{d_{h}}{d_{h}-1}} \quad x \gg 1 .
$$

On the other hand, we expect for small $x$ that

$$
F(x) \sim x^{d_{H}-1},
$$

where $d_{H}$ is another Hausdorff dimension characterizing the quantum geometry at short distances, and is related to the anomalous dimension $\eta$. Measurements of $G_{V}(r)$ have been performed for systems with a variety of matter types. A simple way to determine the cosmological Hausdorff dimension $d_{h}$ is to plot $G_{V}(r)$ against $r$. Even if the function $F(x)$ is not known explicitly, one can obtain an estimate of $d_{h}$ by comparing the maxima of these curves for different volumes $V$. One then plots the rescaled quantities $G_{V}(r) / V^{1-1 / d_{h}}$ as a function of $x$, where according to (112) the curves for different volumes $V$ should fall on top of each other. This procedure can be viewed as a finite-size scaling method, of the type used in the study of critical phenomena in statistical physics. In the context of quantum gravity, it was introduced in refs. [50,51] for $d=2$, and in refs. [52, 53] for $d=4$.

For pure two-dimensional gravity one can compute $F(x)$ exactly, and finds $d_{H}=d_{h}=4$. Comparing this to the numerical analysis, it was discovered that a scaling form with the correct value of $d_{h}$ can only be obtained by using a shifted scaling variable defined by

$$
x_{\delta}=\frac{r+\delta}{V^{1 / 4}}
$$

where the constant $\delta$ is determined numerically. In the case of pure gravity the shift $\delta$ can be calculated analytically, but for more complicated systems this is usually not the case. The agreement between theory and numerical simulations is impressive. In Fig. 7 we show the scaling of $G_{V}(r)$ for various volumes $V$ and the theoretical curve (which can hardly be seen since it coincides perfectly with the numerical data). 


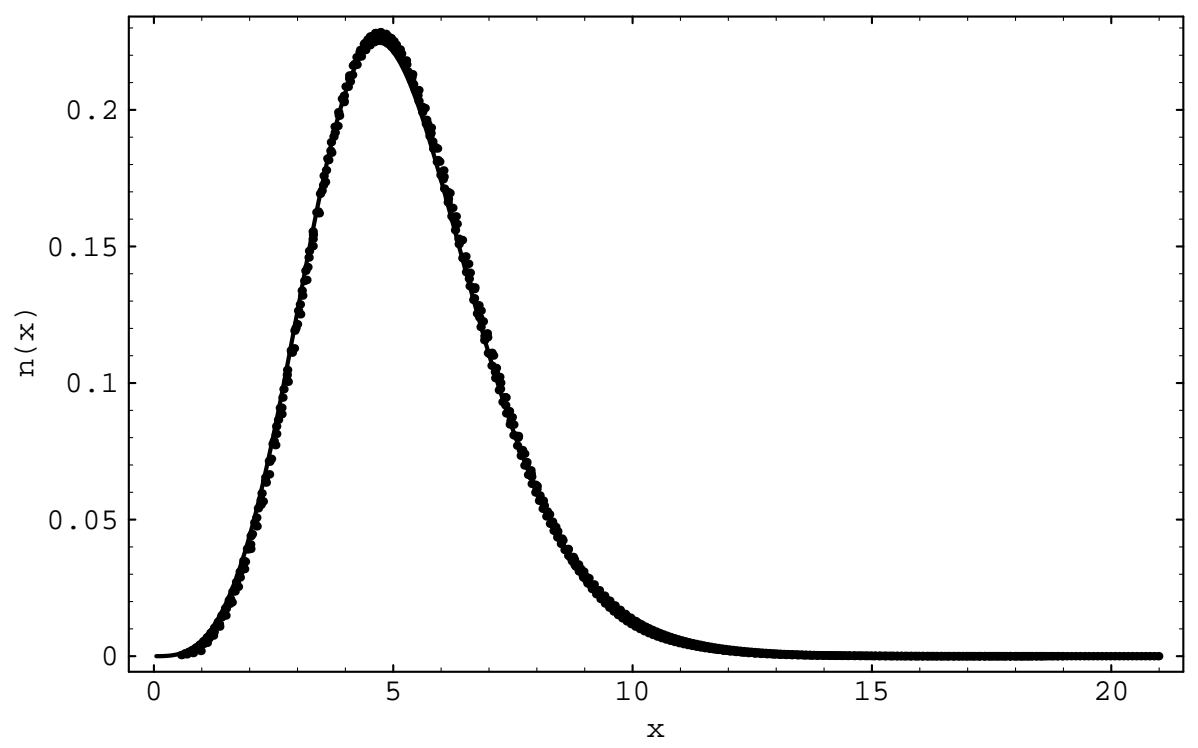

Figure 7. The distributions $n(x) \equiv G_{V}(x) / V^{1-1 / d_{h}}$ in 2 d Euclidean gravity, for volumes $V=1000,2000,4000,8000,16000$ and 32000 , together with the theoretical distribution $F(x)$.

Such a shift is also necessary in more complicated systems. It was soon realized that for the case of pure gravity this shift is a leading-order finitesize correction to the scaling form (112), which was obtained for $V \rightarrow \infty$. This improved scaling could also be used to check the small- $x$ behaviour of $F(x)$ with very good accuracy.

Another important test of our numerical methods is to check whether the scaling limit of the two-point function is indeed independent of the detailed definition of the distance function. Numerically it is easy to measure the two-point function $\bar{G}_{V}(r)$, where $r$ is now the link distance on the triangulation. In this case we have no exact analytical prediction, but we may again fit the numerical results (which look very similar to those for $G_{V}(r)$ ) to the scaling relation (112). After a trivial rescaling of the distance, the matching with the theoretical curve $F(x)$ is even better than previously, but again one must include a shift $\bar{\delta}$. Numerically, the shift is much smaller than before. This was to be expected, since a shift in $x$ compensates for small-distance artifacts, which are larger on a lattice with smaller vertex valence. The aspects mentioned above are illustrated in Fig. \& by data obtained in the study of a $c=-2$ conformal field theory coupled to $2 \mathrm{~d}$ 

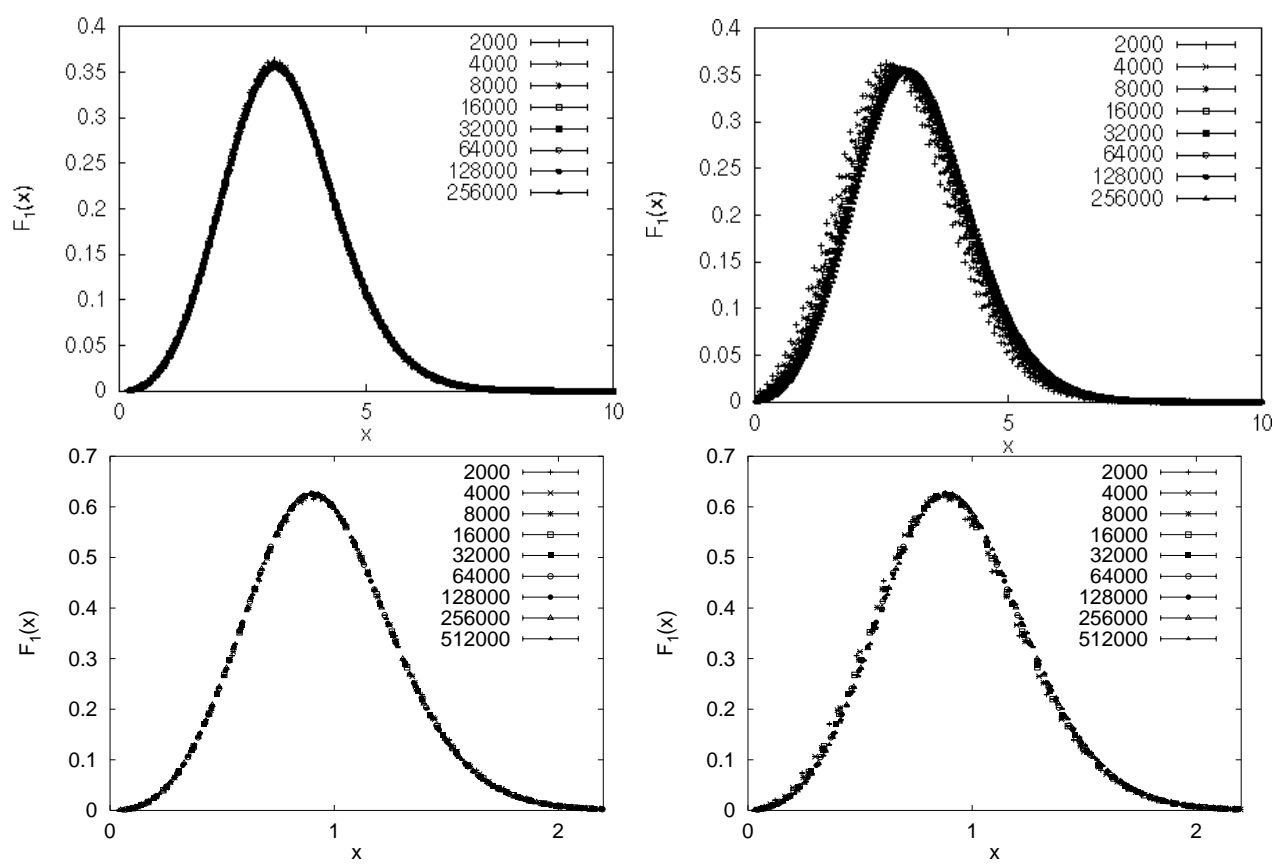

Figure 8. The two upper figures depict the measured distributions $G_{V}(r)$ of $2 \mathrm{~d}$ Euclidean gravity coupled to $c=-2$ conformal matter, defined in terms of the dual link distance and rescaled according to eq. (112) to get $F(x)$, with a shift $\delta=4.5$ (left) and without a shift (right). In the two lower figures, the link distance has been used, again with a shift $\delta=0.5$ (left) and without(right). The best fit for the Hausdorff dimension $d_{h}$ extracted from the data is $3.58 \pm 0.01$, in agreement with theoretical predictions [55].

Euclidean gravity [54].

Analogous results hold for branched-polymer systems, whose two-point functions can be calculated exactly. The distance $r$ is in this case given by the (unique) number of links separating each pair of points. For fixed volume $V \rightarrow \infty$, we find a universal scaling of the form (112), but now with an (intrinsic) Hausdorff dimension $d_{h}=2$, which also coincides with the value for $d_{H}$. Again a shift in $x$ is needed as a leading finite-size correction to obtain optimal agreement with the theoretical results.

To summarize, measuring $G_{V}(r)$ or $\bar{G}_{V}(r)$ provides an efficient way for determining the Hausdorff dimension $d_{h}$, also in cases where the two-point functions are not known exactly. To take care of short-distance effects, it is important to work with an improved, shifted scaling variable $x_{\delta}$. In practice one measures $G_{V}(r)$ for a number of volumes $V$, and tries to find a best fit to the universal scaling relation (112) by fitting both $\delta$ and $d_{h}$.

Another geometric observable characterizing a compact metric manifold is its spectral dimension, which is related to the diffusion equation and the 
spectrum of the Laplace operator. If a diffusion process is started with a completely localized initial condition, one can measure the return probability $P(T)$ of a fictitious test particle after "time" $T$. On a fixed continuum geometry, one obtains after averaging over the initial point that

$$
P(T) \sim \frac{1}{T^{d_{s} / 2}} \sum_{k} a_{k} T^{k}
$$

for small $T$, where the coefficients $a_{k}$ can be expressed in terms of local geometric invariants. We can measure $d_{s}$ also in the discretized theory, but some care must be exercised when comparing with a continuum formula like (117), since it is exactly the short-time limit $T \rightarrow 0$ that is ill-defined in the discrete case. This can already be seen in the simple case of diffusion on a discrete one-dimensional line, with a discrete Laplacian and a continuous time. The diffusion equation

$$
\frac{d \phi_{i}}{d T}=\frac{\phi_{i+1}+\phi_{i-1}-2 \phi_{i}}{2}
$$

with the initial condition $\phi_{i}(0)=\delta_{i 0}$ can be solved exactly, yielding

$$
P(T)=e^{-T} I_{0}(T) \sim \frac{1}{T^{1 / 2}}(1+\mathcal{O}(1 / T)),
$$

where $I_{0}(T)$ is the Bessel function. One therefore rederives the correct spectral dimension $\left(d_{s}=1\right)$ only for large $T$. The short-time behaviour is completely different and dominated by discretization artifacts. For this simple example, one can check numerically the effect of the finite system size, by changing the discrete line to a closed circle with $V$ points. One observes three distinct regions:

- small time $T$, which is similar to the example above, and dominated by discretization effects;

- intermediate $T$, where one obtains the correct value of $d_{s}$; and

- very large $T$, where the system approaches a stationary translationinvariant state.

A similar structure is also expected in less trivial cases, such as simplicial quantum gravity in two dimensions and higher. This behaviour is illustrated in Fig. 9, where we show the results of measuring the spectral dimension for 2d Euclidean quantum gravity coupled to matter fields of various central charges $c$.

The measurement of the spectral dimension in $2 \mathrm{~d}$ Euclidean quantum gravity is a nice illustration of the fruitful interaction between numerical "experiment" and theory. Since the quantum geometry of $2 \mathrm{~d}$ Euclidean 


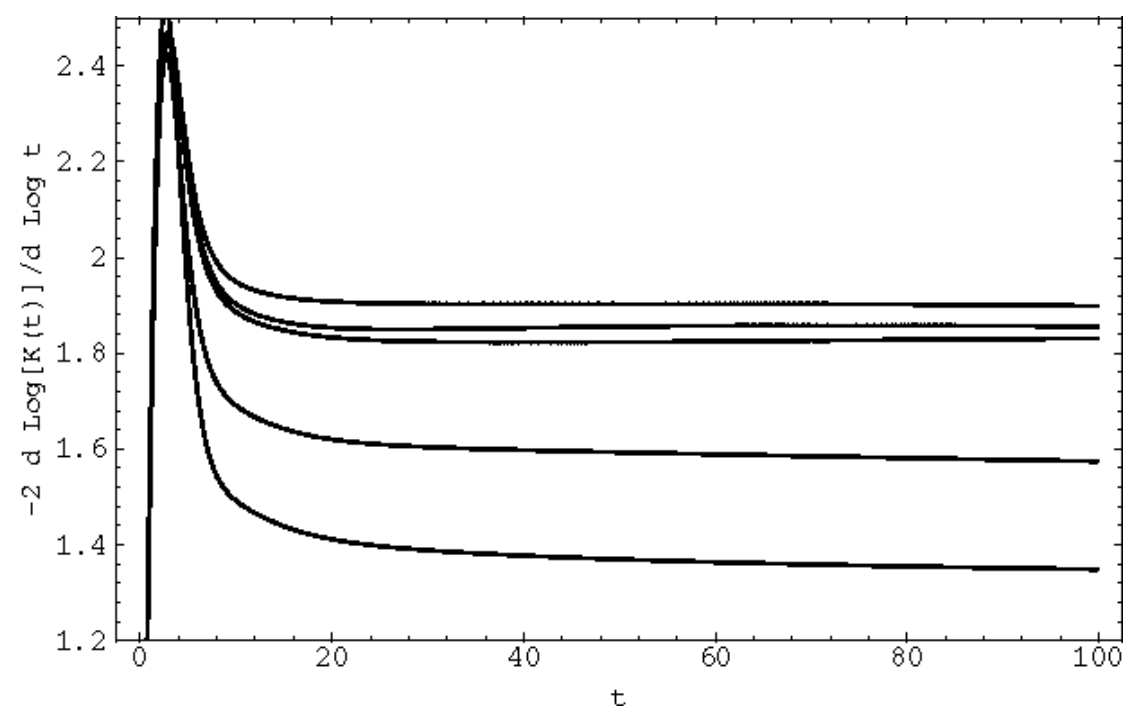

Figure 9. The spectral dimension $d_{s} \approx-2 d \log P(T) / d \log T$ versus $T$ for $c=0$ (top curve), $c=1 / 2, c=1, c=3$ and $c=5$ (bottom curve) theories coupled to $2 \mathrm{~d}$ Euclidean quantum gravity. The system size is $V=16000$ triangles.

gravity has a fractal Hausdorff dimension $d_{h}=4$, one could also have expected an anomalous spectral dimension $d_{s}$. However, numerical simulations consistently found that $d_{s}=2$, no matter which field theory was coupled to gravity, as long as the central charge $c$ remained $\leq 1$. This inspired an analytic proof of this value, using continuum conformal Liouville theory and an assumption about finite-size scaling [56, 57]. We should point out that $d_{s}=2$ does not imply that one cannot see the fractal structure corresponding to $d_{h}=4$ in the diffusion process. It manifests itself in the relation

$$
\langle R\rangle_{T} \sim T^{1 / d_{h}}
$$

between the "time" $T$ and the average geodesic distance $R$ diffused in that time. We refer again to $[56,57]$ for details.

For $c>1$, the numerical estimate was close to $d_{s}=4 / 3$. Eventually it was proven that generic branched polymers have a spectral dimension $d_{s}=$ $4 / 3$ [58], thus reconciling theory and numerical results. At the same time, it provided independent evidence that for $c>1$ the space-time degenerates into branched polymers.

We have so far discussed only purely geometric observables. For theories coupled to matter, there will be additional critical exponents characterizing the behaviour of the system close to the critical matter coupling $\beta_{c}$. Near this transition point, we expect to find a divergent correlation length $\xi(\beta) \sim\left|\beta-\beta_{c}\right|^{-\nu}$, for some positive $\nu$. Since we always study systems of 
finite size, we will never observe a genuine phase transition, but merely a pseudo-critical point $\beta_{c}^{*}(V)$, where the linear extension $L$ of the system behaves like $L \sim \xi(\beta)$. For a finite system $L^{d}=V$. A system with a dynamical geometry may possess two scales: one associated with the cosmological constant when $\mu \rightarrow \mu_{0}$ and another one related to the matter phase transition. Using standard finite-size scaling arguments, we expect that the measured values of the specific heat $c_{v}$, the magnetization per unit volume $m$, and the magnetic susceptibility $\chi$ at the pseudo-critical point $\beta_{c}^{*}$ behave like

$$
\begin{aligned}
c_{v}\left(\beta_{c}^{*}\right) & \sim V^{\frac{\alpha}{\nu d}}, \\
m\left(\beta_{c}^{*}\right) & \sim V^{-\frac{\beta_{m}}{\nu d}}, \\
\chi\left(\beta_{c}^{*}\right) & \sim V^{\frac{\gamma m}{\nu d}},
\end{aligned}
$$

where $\alpha, \beta_{m}$ and $\gamma_{m}$ satisfy the scaling relations

$$
\alpha+2 \beta_{m}+\gamma_{m}=2, \quad 2 \beta_{m}+\gamma_{m}=\nu d .
$$

For magnetic systems corresponding to $0<c<1$, one knows from analytical calculations the values of the critical exponents as well as the values of the critical points $\beta_{c}$.

A relation between the scalings in the geometric and matter sectors can be obtained by generalizing the two-point functions $G_{\mu}(r)$ and $G_{V}(r)$, to include also correlations between the matter fields at geodesic distance $r$. One example of a quantity of this kind is

$$
G_{\mu}^{\{\phi, \psi\}}(r)=\sum_{\tilde{\mathcal{T}}} \sum_{\phi_{i}} e^{-\mu N_{2}-S_{\text {matter }}\left(\phi_{i}\right)} \sum_{i, j} \phi_{i} \psi_{j} \delta_{D(i, j), r},
$$

which may be expressed as the discrete Laplace transform of a finite-volume correlator,

$$
G_{\mu}^{\{\phi, \psi\}}(r)=\sum_{V} e^{-\mu V} G_{V}^{\{\phi, \psi\}}(r) .
$$

In this expression, $\phi_{i}$ and $\psi_{j}$ are local functions of the matter or geometric variables at the points $i$ and $j$. A typical example is the spin-spin correlation function of the Ising system. At the combined critical point of the Ising model and the cosmological constant, (123) yields a reparameterizationinvariant definition of a matter correlator in quantum gravity. More precisely, the correlation function is that of a $c=1 / 2$ conformal field theory coupled to $2 \mathrm{~d}$ Euclidean quantum gravity. Note also that the previously defined geometric two-point function (106) is a special case of (123), with the unit operator replacing the spin operators.

This construction has provided us with a new understanding of the KPZ-exponents of conformal field theories coupled to gravity. These exponents may be viewed as the "dressed" scaling exponents of the primary 


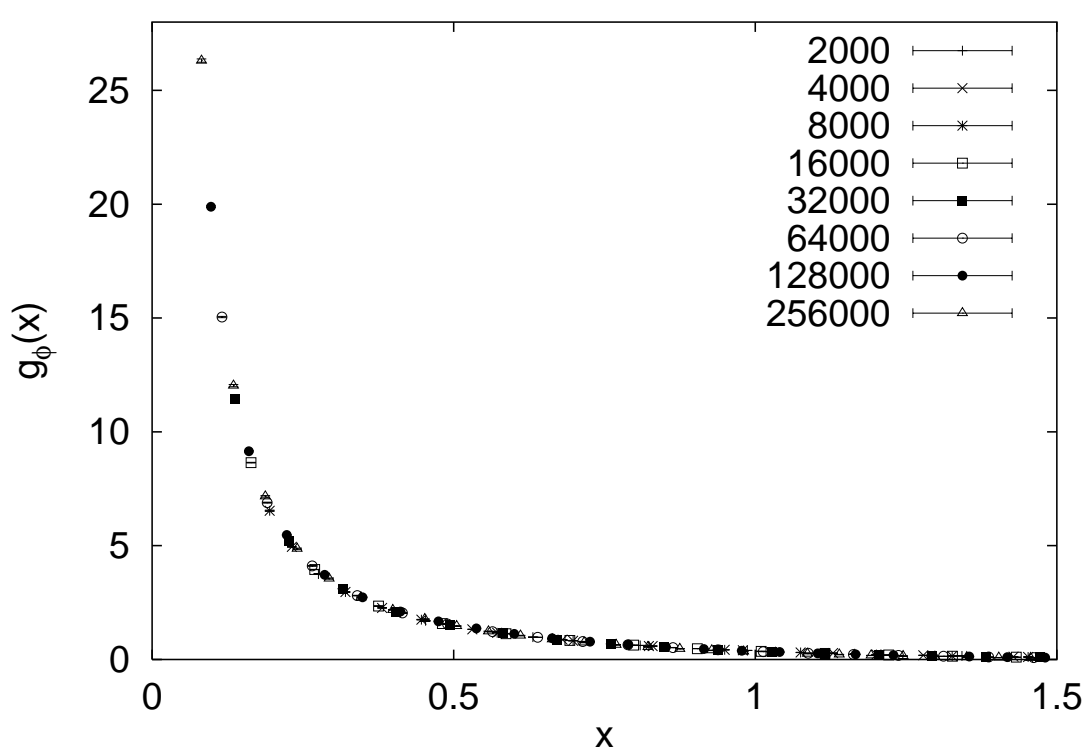

Figure 10. The normalized spin-spin correlation function for the Ising model coupled to $2 \mathrm{~d}$ Euclidean gravity, plotted as a function of the dimensionless length $x=r / N_{T}^{1 / d_{h}}$, where $r$ is the geodesic length and $N_{T}$ the space-time volume. The number of triangles $N_{T}$ ranges from 2000 to 256000 and the Hausdorff dimension is $d_{h}=4.0$. The short-distance behaviour is in accordance with KPZ scaling.

fields in the conformal theory. However, before it was realized that they should be analyzed in terms of the definition (123), it was not clear how to generalize the usual flat-space correlators because of the requirement of reparametrization-invariance (see [59, 60] for further discussion). Fig. 10 shows the result of a numerical simulation of the (suitably normalized) spin-spin correlator.

We should point out a subtlety in the definition of the correlator (123). If the expectation values of the fields entering are not zero, one might be interested in defining the corresponding connected correlator. However, there is no straightforward way of defining such an object since (123), in addition to the usual average over field configurations, also includes an average over geometry. A discussion of possible definitions of the connected part of the two-point correlators and their scaling properties can be found in [61].

All of the observables introduced above can also be used in numerical simulations of higher-dimensional gravity-matter systems, and - apart from the ones involving baby-universe counting - also for Lorentzian quantum gravity. 


\subsection{COMMENTS ON THE 2D RESULTS}

Our obvious starting point in 2d Euclidean quantum gravity was the measurement of $\gamma_{s t r}$, in order to compare it with the theoretical prediction for $c<1$, namely,

$$
\gamma_{s t r}=\frac{c-1-\sqrt{(c-1)(c-25)}}{12} .
$$

For $c>1$, this quantity becomes imaginary and its interpretation in terms of Liouville theory breaks down. For $c=1$, logarithmic corrections appear. For a number of special values of $c$, we have explicit discrete models, which at their critical points represent conformal field theories with charge $c$. They are pure 2 d gravity $\left(c=0, \gamma_{s t r}=-1 / 2\right)$, the Ising model $\left(c=1 / 2, \gamma_{s t r}=\right.$ $-1 / 3)$, the 3 -state Potts model $\left(c=4 / 5, \gamma_{s t r}=-1 / 5\right)$, the 4 -state Potts model $\left(c=1, \gamma_{s t r}=0\right)$, and a single massless scalar field $\left(c=1, \gamma_{s t r}=0\right)$. In all of these cases, formula (125) has been verified with great accuracy. A coupling of several matter fields corresponds to adding their central charges.

Away from their critical points $\beta_{c}$, the finite spin systems approach a pure-gravity behaviour, with $\gamma_{s t r} \rightarrow-1 / 2$. This observation can be turned into a method for locating $\beta_{c}$, by monitoring the change in $\gamma_{s t r}$ as the coupling $\beta$ is varied (Fig. 11).

For all the spin systems, measurements of the magnetic critical exponents defined in (121) are in perfect agreement with the theoretical predictions . We conclude that for the matter models with $0 \leq c \leq 1$ which have been used in numerical simulations, the scaling limit of the discretized theory corresponds to a unitary matter model coupled to Liouville gravity.

A somewhat surprising result in this range of $c$ is the numerical value of $d_{h}$ obtained from the scaling of $G_{V}(r)$. For $0 \leq c<1$, there are two theoretical predictions for $d_{h}$, namely

$$
d_{h}^{(i)}=2 \frac{\sqrt{25-c}+\sqrt{49-c}}{\sqrt{25-c}+\sqrt{1-c}}
$$

derived by studying diffusion in a fluctuating geometry, using Liouville theory [55], and

$$
d_{h}^{(i i)}=\frac{24}{\sqrt{1-c}(\sqrt{1-c}+\sqrt{25-c})}
$$

\footnotetext{
${ }^{7}$ This also includes the case $c=1$, which can be realized either as two Ising spins, as a $q=4$ Potts model, or as a single Gaussian field coupled to gravity. However, there are logarithmic corrections to formula (102) which have to be included in the fits in order to obtain $\gamma_{s t r}=0$ from the data.
} 


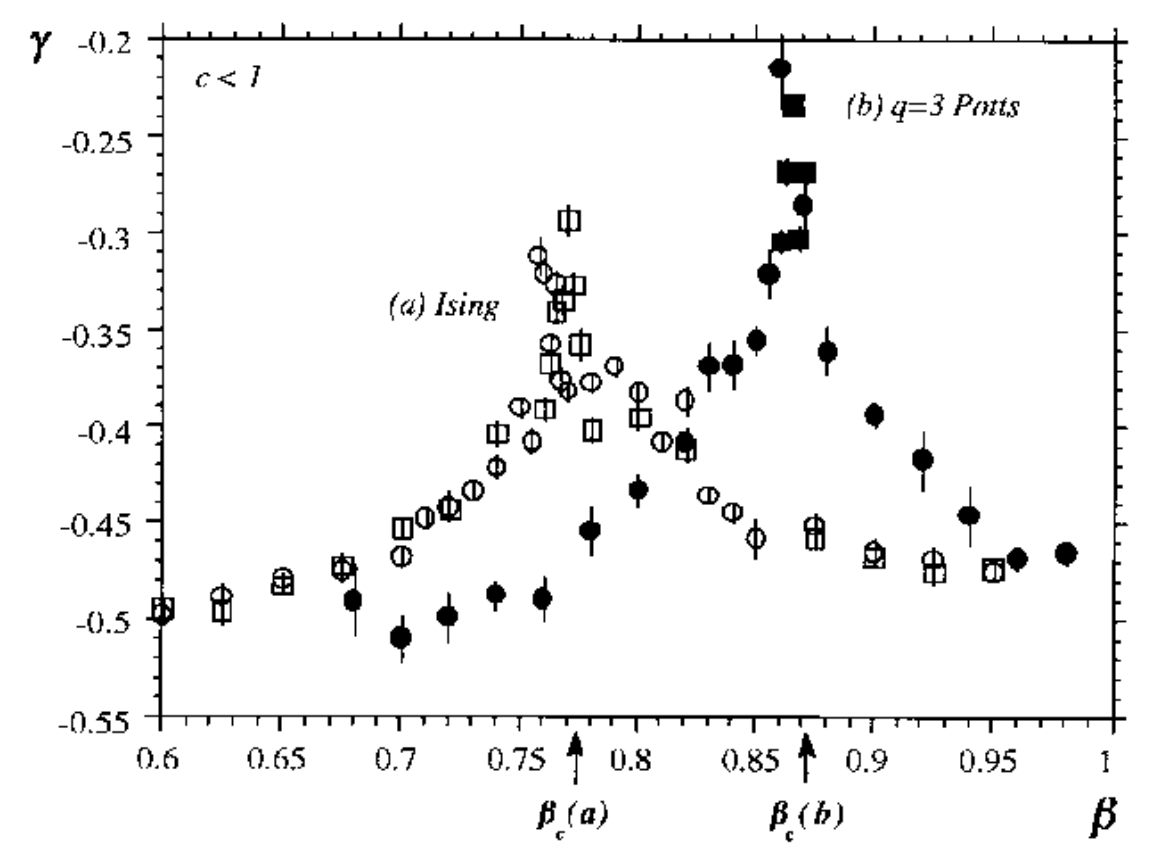

Figure 11. Measurements of $\gamma_{s t r}$ as a function of $\beta$, for the Ising model (white), and the 3 -state Potts model (black), at system sizes $N=1000$ (circles) and 2000 (squares).

derived from matrix model considerations [62]. The formulas agree for $c=0$ (where $d_{h}=4$ ), but differ elsewhere. In both derivations there are plausible, but unjustified, assumptions. Neither of them could be confirmed by numerical simulations 8 . Instead, a numerical estimate $d_{h} \simeq 4$ was obtained for all cases with $0 \leq c \leq 1[51,59]$ together with $d_{H} \simeq d_{h}[60,59]$ from the small- $x$ behaviour of $F(x)$. The numerical errors seem sufficiently small to support the conjecture that $d_{h}=4$ for unitary matter theories coupled to $2 \mathrm{~d}$ gravity. The spin-spin correlation functions measured for the Ising system $(c=1 / 2)$ at $\beta=\beta_{c}$ seem to indicate that the matter degrees of freedom do not introduce a new scale and that also for these correlations, there is a universal scaling corresponding to $d_{h} \simeq 4$.

Our numerical information for the case $c<0$ is only partial. A special case is $c=-2$, where the simulations can be based on a direct generation of diagrams, instead of Monte Carlo methods. As already mentioned in connection with Fig. 8 this led to $d_{h}=3.58 \pm 0.02$, which is very close to

\footnotetext{
${ }^{8}$ It is now understood that formula $(127)$ measures the fractal dimension of (generalized) spin clusters of the matter fields, rather than the fractal dimension of the underlying geometry [63].
} 
the value of $d_{h}=3.561 \ldots$ predicted by $(126)$. Less conclusive numerical data for $c=-5$ also show agreement with (126). Formula (126) also predicts that $d_{h}=2$ as $c \rightarrow-\infty$, which is the expected flat-space behaviour. It thus seems that eq. (126) could be correct for $c \leq 0$. It is still not understood why the formula fails for $0<c \leq 1$.

For $c>1$, the minbu size diverges as $V \rightarrow \infty$. Systems with $c>1$ can easily be studied numerically by coupling several copies of spins or scalar fields. In all cases studied, $\gamma_{s t r}$ was found to be positive and to approach $+1 / 2$ for $c \simeq 4$ and bigger, suggestive of a branched-polymer system. This interpretation would also agree with the scaling analysis of $G_{V}(r)$ which is consistent with $d_{h} \rightarrow 2$, and the measurement of $d_{s}$, consistent with $d_{s} \rightarrow 4 / 3$. It is not clear to what extent the observed behaviour is universal, since the numerical estimates depend on the details of the regularization. For example, the approach to the branched-polymer phase seems slower when regular triangulations $T$ rather than generalized configurations in $\mathcal{T}$ are used. This could be due to finite-size effects, or alternatively to the presence of other universality classes of branched-polymer systems.

In the 2d Euclidean case, we have learned from both theoretical and numerical investigations that there is only a small "window" of physically sensible theories, by which we mean unitary matter models coupled to gravity. If we move outside this range by adding too many matter fields, the theory breaks down. This indicates that matter and geometry are interacting strongly.

Baby universes play a crucial role in understanding the nature of the spin-gravity interaction, highlighting at the same time the difference between the Euclidean and Lorentzian gravity models. Again the Ising model serves as an ideal illustration. The Ising ground state at zero temperature, for both fixed and fluctuating geometries, is the state where all spins are aligned. Since the energy of a given spin configuration is proportional to the length of the boundary separating spin-up and spin-down regions, the dominant spin configurations at low temperature (equivalently, at large spin coupling) are those with minimal spin boundary lengths.

The lowest spin excitations contributing to the free energy density come from spin clusters with boundaries of minimal length. Unlike on flat, regular lattices, a short spin boundary does not imply that there are few spins inside. On the contrary, the typical situation in Euclidean quantum gravity has an entire baby universe of up-spins, say, on one side of the minimal boundary, and the parent universe with opposite spin orientation on the other, as illustrated in Fig. 12. Since the baby universe can have any size, there is no restriction on the size of spin clusters, even at low temperature. The introduction of a fluctuating geometry with baby universes thus has a strong effect on the matter behaviour. In turn, since short spin boundaries 


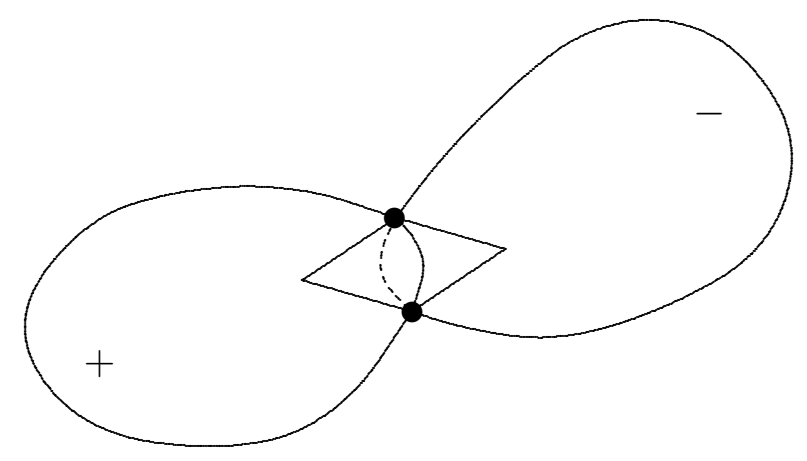

Figure 12. Two spin clusters separated by only two links which form a "bottleneck" on the surface. Two of the four triangles containing one of the two spin-boundary links are also shown.
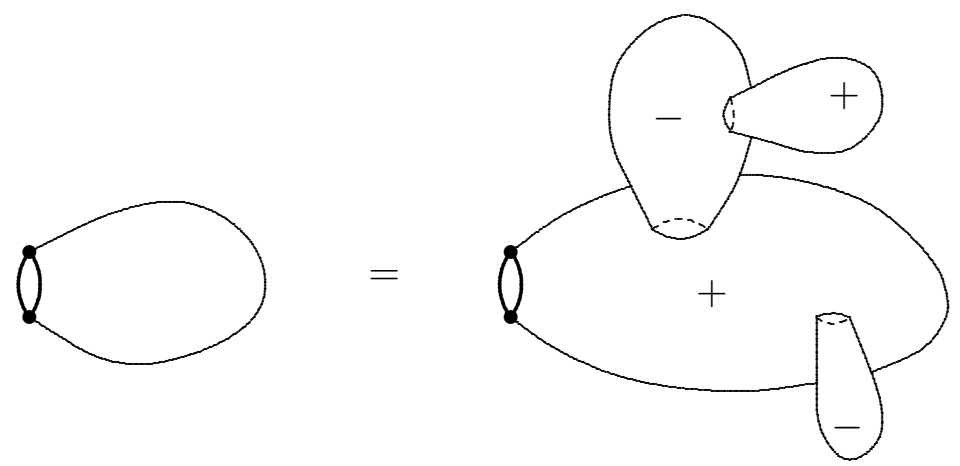

Figure 13. A surface with a minimal boundary and its recursive decomposition into baby universes associated with spin clusters.

are energetically preferred, the matter has a tendency to "squeeze off" the underlying geometry, generating even more baby universes.

When more than two Ising models are coupled to $2 \mathrm{~d}$ Euclidean quantum gravity, the matter-geometry interaction becomes so strong that the geometries degenerate into so-called branched polymers, which can be viewed as infinitely branched trees of baby universes of cut-off size (the lattice spacing a). This provides us with an explicit picture of the $c=1$ barrier of $2 \mathrm{~d}$ Euclidean quantum gravity. The proliferation of baby universes and its relation to spin clusters are illustrated in Fig. 13.

Since baby universes are absent from Lorentzian gravity, its interaction with matter is much weaker. The Ising model on Lorentzian geometries has not yet been solved analytically, but its critical exponents have been determined both by a high-temperature expansion and Monte Carlo techniques [12]. The Hausdorff dimension of space-time is still $d_{h}=2$, and the critical 

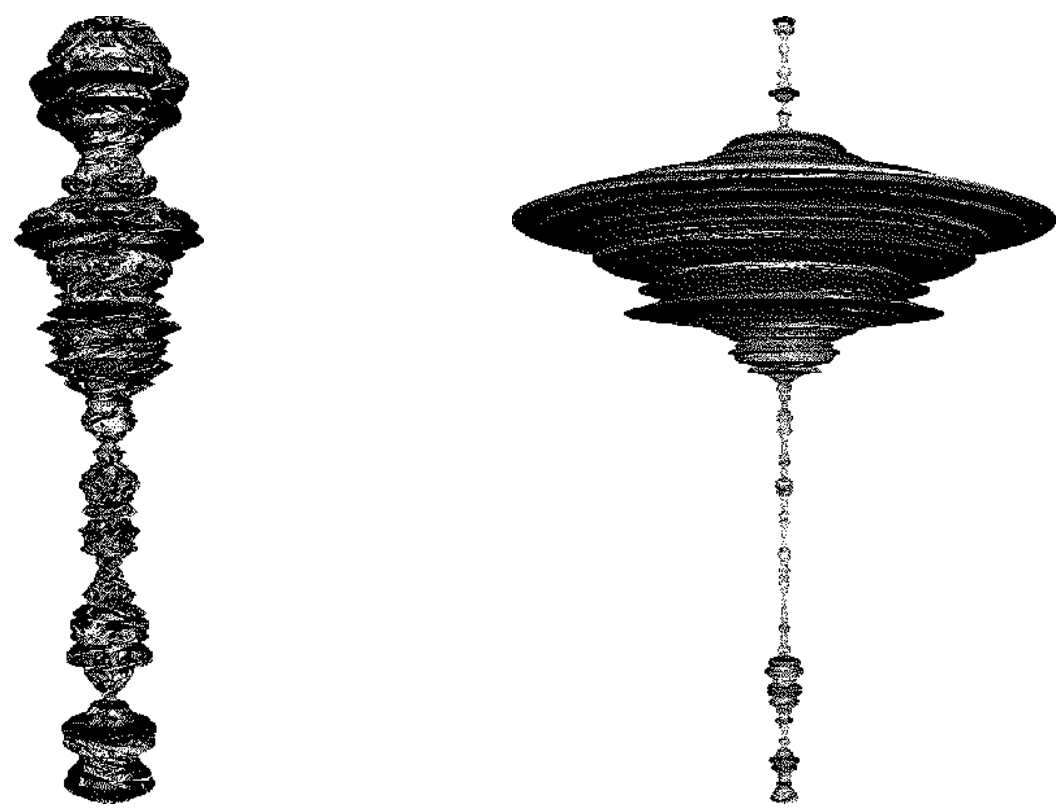

Figure 14. Two typical space-time configurations, resulting from the coupling of one Ising model (left) and eight Ising models (right) to Lorentzian gravity.

Ising exponents retain the Onsager values found on fixed, regular lattices, in spite of large fluctuations of the geometry. It has also been shown [28] that a particular dimer mode 9 coupled to Lorentzian gravity does not change the fractal dimension of the geometry. It is thus tempting to conjecture that the (fractal) dimension of space-time remains equal to 2 as long as the central change of the matter fields is less than or equal to 1.

It is an interesting question whether there is an analogue of the $c=1$ barrier when sufficiently many matter degrees of freedom (with a sufficiently large central charge $c$ ) are added. This is indeed what seems to happen. A phase transition in the geometry has been observed when coupling 8 Ising spin copies (corresponding to $c=4$ ) to 2 d Lorentzian quantum gravity [13]. This effect is illustrated in Fig. 14, where we show typical configurations of the fluctuating Lorentzian geometry in the case of coupling to a single Ising model and to 8 Ising models. The one-Ising triangulation is qualitatively very similar to the fluctuating geometry without any Ising spins, but in the case of 8 Ising spins, the effect of the matter is very pronounced. Since the creation of baby-universe branches is by definition forbidden, the only

\footnotetext{
${ }^{9}$ It is known that the critical behaviour of dimer models is associated with a $c=$ -2 conformal field theory. Since the dimer model considered in [28] imposes certain restrictions on the allowed dimer positions, it strictly speaking has not been proven to lie in the same universality class.
} 
way for the matter to create short spin-boundaries is by squeezing individual spatial slices to a minimal (cut-off) size, producing the long, stalk-like structure seen in Fig. 14. In the remainder of space-time, the spatial slices attain a macroscopic size. On this extended part of the universe, the scaling of the average spatial length in terms of the cosmological constant is anomalous,

$$
\langle L\rangle_{\Lambda} \sim \frac{1}{\Lambda^{2 / 3}},
$$

which was not the case for $c \leq 1$. Moreover, the critical Ising exponents, when measured on the extended region, are still given by the Onsager values. We therefore have identified an analogue of the $c=1$ barrier, at which

a geometric phase transition takes place. However, its effect is much milder than in Euclidean gravity. Ising-life continues even beyond the barrier. This underlines yet again the drastic difference between $2 \mathrm{~d}$ gravity with and without baby universes.

\section{Dynamically triangulated quantum gravity in $d>2$}

\subsection{GENERALIZATION TO HIGHER DIMENSIONS}

The general outline of the simplicial regularization of Euclidean quantum gravity presented above remains true in dimension $d>2$, where the fundamental building blocks are $d$-simplices with $d+1$ vertices. The partition function for dynamically triangulated pure gravity is given by

$$
Z=\sum_{\bar{T}} \frac{1}{N_{0}(T) !} e^{-S_{E H}},
$$

where the sum is taken over a class of labelled $d$-dimensional triangulations with spherical topology. In the following, we will be mainly interested in the cases $d=3,4$, where the discretized Einstein-Hilbert action (87) can be written equivalently as

$$
S_{E H}=\kappa_{d} N_{d}-\kappa_{0} N_{0},
$$

using the Dehn-Sommerville relations. Since for simplicial manifolds and fixed volume $N_{d}$, the number of vertices has an upper limit $N_{0} \propto N_{d} / d$, the action (130) is bounded for finite volume.

Note that in $d>2$ it is not possible to realize a locally flat geometry by gluing together equilateral simplices. Only in $2 \mathrm{~d}$ there is a regular tiling of flat space, in which each vertex is shared by six triangles. This happens because the dihedral angle $\theta_{d}$ of each $d$-simplex contributing to the parallel transport around a $(d-2)$-simplex satisfies $\theta_{d}=\arccos 1 / d$, which for $d>2$ 
is not a rational fraction of $2 \pi$. However, this is a short-distance property which should become irrelevant in the scaling limit.

In the simplest case of gravity without matter, there are two couplings: $\kappa_{d}$, which is proportional to the cosmological constant, and $\kappa_{0}$, related to the gravitational constant. Both quantities are dimensionless in the lattice formulation, but will get their dimension back through "dimensional transmutation" in the scaling limit, if it exists.

The gravitational state sum is now

$$
Z=\sum_{V} e^{-\kappa_{d} V} Z_{V}\left(\kappa_{0}\right),
$$

where $V=N_{d}$, and $Z_{V}\left(\kappa_{0}\right)$ is the partition function at fixed volume. In order for the sum (131) to be well defined, $Z_{V}\left(\kappa_{0}\right)$ must be exponentially bounded for large $V$,

$$
Z_{V}\left(\kappa_{0}\right) \sim e^{\kappa_{d}^{c}\left(\kappa_{0}\right) V} z_{V}\left(\kappa_{0}\right) .
$$

The existence of such a bound has been shown [24, 25], but the form of the subleading terms is at present unknown. A power behaviour of $z_{V}$ leads to a situation similar to (102), but there could in principle also be a logarithmic dependence $\log z_{V} \sim V^{\alpha}$, with $\alpha<1$. For systems with a fixed volume one can replace $Z_{V}$ by $z_{V}$ when averaging over configurations. Note that higher-dimensional gravity with the action (130) resembles a spin system in $2 \mathrm{~d}$, with the role of the matter coupling $\beta$ played by $\kappa_{0}$. The cosmological constant will undergo a renormalization according to

$$
Z=\sum_{V} e^{-\left(\kappa_{d}-\kappa_{d}^{c}\right) V} z_{V}\left(\kappa_{0}\right),
$$

and $1 /\left(\kappa_{d}-\kappa_{d}^{c}\right)$ gives an estimate of $\langle V\rangle_{\kappa_{d}}$.

To understand the critical properties of the system defined by (133), one must investigate the limit $V \rightarrow \infty$. For large positive $\kappa_{0}$, configurations with many vertices will be favoured. Comparing with the $2 \mathrm{~d}$ case, we expect this limit to correspond to a branched-polymer phase. Conversely, for large negative $\kappa_{0}$, the system will move towards lower $N_{0}$, leading to "crumpled" configurations. Note that unlike in $2 \mathrm{~d}, N_{0}$ and $V$ are independent, and $N_{0}$ can be very small, for example, it is easy to find triangulations such that $N_{0} \sim V^{a}$ with $a<1$. The fraction $p\left(\kappa_{0}\right)=d\left\langle N_{0}\right\rangle_{V} / V$ can be used as an order parameter of the theory, and is related to the average local curvature. It may vary between 0 and 1 , corresponding to the two extreme geometric phases. In the limit $p=0$ ( $\kappa_{0}$ small $)$, where the geometry is "crumpled", there are only a few vertices and one can move from one to another in only a few steps. The Hausdorff dimension of such geometries is large. In the 
limit $p=1$ (large $\kappa_{0}$ ), the number of vertices for a given $V$ is maximal, leading to branched-polymer configurations with Hausdorff dimension two.

Neither of these two limits looks like a promising candidate for a scaling $d$-dimensional quantum theory. Let us compare this situation with that of a $2 \mathrm{~d}$ spin system. The two extreme phases correspond to the high- and lowtemperature regions of the spin model, where also the spin system has no continuum limit. In the high-temperature limit, the spins fluctuate totally randomly, whereas in the low-temperature limit they hardly fluctuate at all. However, there is a critical temperature $T_{c}$ where the spin fluctuations become critical and long-ranged. At $T_{c}$, one can take a continuum limit and recover a $c=1 / 2$ conformal field theory. In a similar way one might hope to find a critical gravitational coupling $\kappa_{0}$, where a transition between the two phases of geometries takes place, and where more "reasonable" geometries, exhibiting long-range fluctuations, can be observed. Such a point could serve as a non-perturbative fixed point where a theory of quantum gravity can be defined.

Although the problem of solving higher-dimensional gravity with the simple action (130) is a combinatorial problem which superficially looks quite similar to the $2 \mathrm{~d}$ case, we still have no hints of how to find an exact solution. There is a mean-field analysis $[25,26]$ which gives a surprisingly precise description of the phase transition (see also [27]), but most of our understanding of Euclidean dynamical triangulations in $d>2$ comes from performing Monte Carlo simulations, along the lines described in Section 5. Also in these cases a set of ergodic moves is known [46]. Recall that in $2 \mathrm{~d}$, one possible set was given by the $(p, q)$-moves, for integer $p, q>0$ and such that $p+q=d+2$. During a $(p, q)$-move, a subcomplex of $p$ simplices is replaced by one of $q$ simplices with the same $(d-1)$-dimensional boundary. This set can be generalized to higher dimensions [64, 65], using the observation that by gluing the two subcomplexes from before and after the move, one obtains the boundary of a $(d+1)$-simplex. The boundary can be divided into two connected parts (a part $X$ and its complement $\bar{X}$ ) in a finite number of ways. Each move corresponds to replacing some $X \rightarrow \bar{X}$. In two dimensions, the boundary is that of a tetrahedron, made of four triangles. It can be divided either into two pairs of two adjacent triangles or into a single triangle and a set of three triangles sharing a vertex of order three.

Performing an analogous construction in $3 \mathrm{~d}$, one obtains $(1,4)$ - and $(2,3)$-moves, together with their inverses $(4,1)$ and $(3,2)$. The first move inserts a new vertex of order four at the centre of a tetrahedron, thereby creating four new tetrahedra. A $(2,3)$-move takes two tetrahedra which share a triangle, removes this triangle and replaces the two tetrahedra by three tetrahedra, whose common axis (a link of order three) is dual to the 

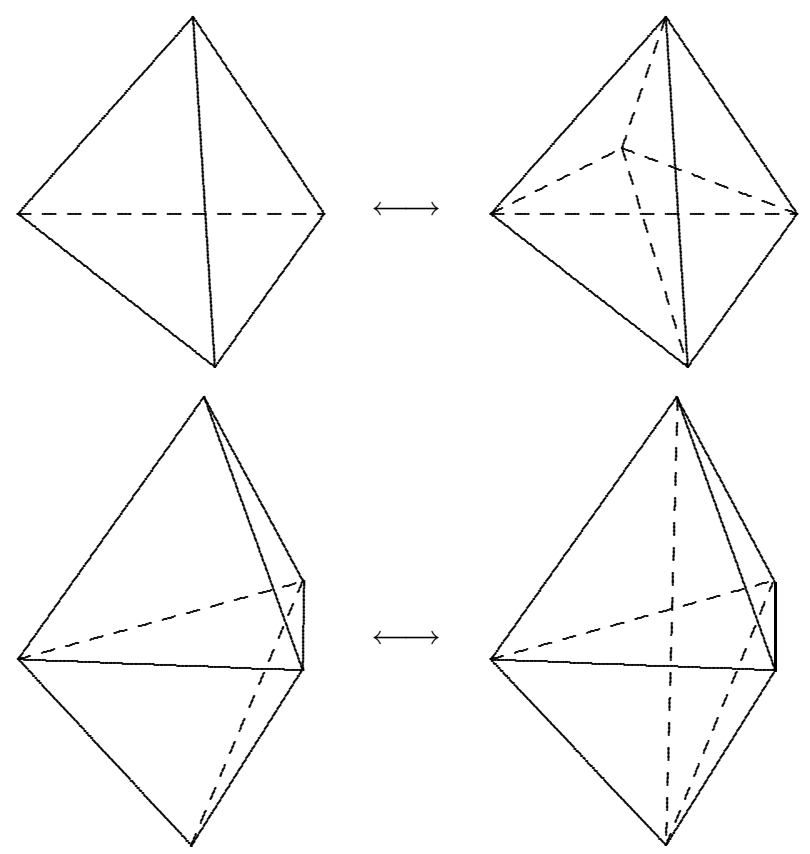

Figure 15. The elementary ergodic moves in $d=3$. There are four different moves since the moves are different from their inverses.

removed triangle. Both types of moves are shown in Fig. 15. A similar construction in $4 \mathrm{~d}$ leads to a set of five $(p, q)$-moves.

These finite sets of moves are ergodic, that is, by repeating local moves, all configurations in the ensemble can be reached. Unlike in $2 \mathrm{~d}$, there are no subsets of moves which are ergodic in the set of triangulations of fixed volume. In $3 \mathrm{~d}$, all $(p, q)$-moves change the volume. If one wants to perform numerical simulations at some fixed $V$, a modification of the procedure used in the simulations becomes necessary.

A simple way of forcing the volume to lie close to a given $V$ is to use a modified action

$$
S_{E H}^{\prime}=\kappa_{d} N_{d}-\kappa_{0} N_{0}+\epsilon\left|N_{d}-V\right|^{\delta}
$$

in the Monte Carlo simulation, typically with $\delta=1$ or 2 , and a free parameter $\epsilon$. The Monte Carlo process now takes place in the set of all triangulations, with fluctuating volume. If we succeed in tuning the cosmological constant to criticality, $\kappa_{d} \approx \kappa_{d}^{c}\left(\kappa_{0}\right)$, the additional term in the action will force the volume to fluctuate around $N_{d}=V$. The amplitude of the fluctuations will depend on the free parameter $\epsilon$, which should be sufficiently small to permit big changes in the geometry. We can then simply collect all configurations with $N_{d}=V$, and use them in measurements, provided they 
are separated by a number of steps much bigger than the autocorrelation time.

This method seems straightforward, but it leads to the following complication. Since our set of moves is ergodic only in the ensemble of simplicial manifolds of fixed topology and of arbitrary volume $N_{d}$, restricting the possible volume fluctuations could make some parts of the configuration space unreachable. For manifolds which are not algorithmically recognizable it can be proven [66] (see also [8]) that the number of steps needed to connect two arbitrary triangulations of identical volume $N_{d}$ cannot be bounded by a computable function $f\left(N_{d}\right)$ (a function which can itself be computed by a finite algorithm). This situation cannot arise when one has a finite set of moves which is ergodic for fixed volume (or even bounded by a computable function), since in this case one can just make a list of all triangulations, which will have a finite, computable length. We conclude that

- if a manifold is not algorithmically recognizable there will be "volume barriers" in the sense explained above, effectively separating parts of the configuration space.

- In a dimension $d$ where algorithmically unrecognizable manifolds exist, one cannot have a single, finite set of volume-preserving moves which at the same time is general, that is, which works for any manifold of the given dimension, and which is ergodic for all manifolds.

In $d=2$ all manifolds are algorithmically recognizable, and the flip move (introduced in Section 5.1) is a volume-preserving move which is ergodic for an arbitrary manifold. It is not known whether there exist three-dimensional manifolds which are not algorithmically recognizable. It has recently been proven that the three-sphere is algorithmically recognizable [67], but it is not known whether the same is true for $S^{4}$. For $d>3$ there exist algorithmically unrecognizable manifolds, and for dimension $d>4$ not even the $d$-sphere is algorithmically recognizable [68].

In the $4 \mathrm{~d}$ numerical simulations we use triangulations with $S^{4}$-topology, and the reliability of the procedure outlined above could depend on the manifold being algorithmically recognizable. If it is not, one might worry that there are configurations, or even large regions which are separated from the rest of the configuration space by very high "volume barriers". This would imply that in order to reach such a configuration by a successive application of the elementary moves, one would be forced to go through very large intermediate volumes.

Attempts have been made to detect such barriers in numerical simulations on $S^{4}$, but without success [69]. Unfortunately, this cannot be taken as an indication that $S^{4}$ is algorithmically recognizable because even for the five-sphere $S^{5}$, which is known to not have this property, such barriers have not been seen [70]. There are two ways of interpreting the $S^{5}$-result. 
Either the Monte Carlo simulation explores only a fraction of the configuration space (in some sense it does anyway, since one always deals with a finite sample of "typical" configurations), or the configurations "beyond the barrier" which are hard to reach constitute only a very small fraction of the configuration space. For $S^{4}$, there need not be any problems in case it is algorithmically recognizable. If it is not, we hopefully are in a situation where the "unreachable" configurations form only a small subset of configurations that becomes negligible in the infinite-volume limit.

Just as in $2 \mathrm{~d}$, the local moves can be supplemented by minbu surgery moves, using a suitably generalized concept of (minimal) baby universes. They are again defined as parts of the triangulation which are connected to the parent universe by $(d-1)$-dimensional minimal necks, which topologically are boundaries of $d$-simplices. The baby universes again play an important role, especially in the large- $\kappa_{0}$ phase, and using an improved algorithm has been essential in studying the phase structure of the fourdimensional theory.

The observables described in $2 \mathrm{~d}$ also exist for gravity in higher dimensions. The minbu distribution can be measured and used to determine $\gamma_{s t r}$ (in case the behaviour of $z_{V}\left(\kappa_{0}\right)$ is power-like), and the scaling properties of the two-point function $G_{V}(r)$ yield information about the Hausdorff dimension. Matter degrees of freedom can be included without problems, the

simplest being spins and massless scalar fields. The coupling of gauge fields to $4 \mathrm{~d}$ dynamical triangulations will be discussed in the next section. - In all these cases we can employ finite-size scaling and measure the matter correlators.

\subsection{NUMERICAL RESULTS IN HIGHER DIMENSIONS}

The algorithm outlined in the previous section was first applied to pure 3d gravity [71]. As predicted, both a branched-polymer and a crumpled phase were discovered, but the transition between them turned out to be a very strong first-order phase transition. This manifests itself in a very large hysteresis in the order parameter $p\left(\kappa_{0}\right)$, when changing $\kappa_{0}$ across the phase transition point. The numerical effort spent on the $3 \mathrm{~d}$ case was relatively small. Since the phase transition is first-order, it cannot give rise to a scaling limit. The phase structure remained unchanged also when spin fields were added [72].

Soon afterwards a numerical algorithm for $4 \mathrm{~d}$ pure gravity was constructed [73] and its phase structure studied extensively. It looked similar to the one in $3 \mathrm{~d}$, with two distinct phases. However, this time the phase transition was much softer, and for a long time was believed to be continuous. This exciting possibility led to many numerical explorations of the 
properties of the two phases and the nature of the phase transition. It was shown that for large $\kappa_{0}$ one obtains a generic branched-polymer phase with $\gamma_{s t r}=1 / 2$ and $d_{h}=2$. Minbu surgery moves were essential in generating independent configurations in this phase. The structure of the crumpled phase seemed rather unusual: the behaviour of $z_{V}\left(\kappa_{0}\right)$ was no longer dominated by a power of $V$, and the scaling of $G_{V}(r)$ suggested that $d_{h} \rightarrow \infty$. (This implies that the average linear extension of the system is almost independent of $V$.) It was then understood that these pathologies are related to the appearance of exactly two "singular" vertices of very large order $\sim V$. A finite fraction of the simplices of the triangulations shares these two vertices, giving rise to a "volume condensation". The two points are always joined by a link, whose order also grows with the volume [74].

The appearance of these structures is well described both by the meanfield approach mentioned earlier [26], and by a phenomenological, so-called balls-in-boxes model [27]. This model represents the gravitational system as a collection of $N_{0}$ boxes, where the $i$ 'th box contains $q_{i}$ balls, analogous to the order $o(i)$ of a vertex $i$ in the simplicial complex. Note that the vertex orders $o(i)$ on a triangulation satisfy

$$
\sum_{i} o(i)=(d+1) N_{d}
$$

In the simplified model, the $q_{i}$ are required to satisfy the same constraint. The local probability of finding $q$ balls in a box is given by $p(q) \sim q^{-\beta}$, where $\beta$ is a free parameter. The model can be solved exactly in the thermodynamic limit, and exhibits two phases: one in which the balls are distributed randomly in all boxes, and a "crumpled" phase at large $\beta$ where a finite fraction of the balls appears in one box. Note that this model has no concept of neighbouring boxes, and there are no correlations among the $q_{i}$ (other than the relation (135)).

Although the balls-in-boxes model can hardly claim to reveal much about the geometric structure of quantum gravity, it nevertheless seems to capture some important features of the situation observed in the numerical simulations. In particular, one might wonder whether also in the full simplicial-gravity model there are no correlations between the vertex orders. This conjecture can be tested by investigating the corresponding gravitational two-point correlator for vertices separated by a (geodesic) distance $r$. Such a correlator is similar to a matter-matter correlator with non-vanishing expectation values for the matter fields. (We will not discuss how to define correctly its connected part.) Preliminary measurements indicate that the correlations do not extend beyond a few lattice spacings, that is, they are of the order of the cut-off [77] (see also [78] for an earlier report). Similarly short-ranged correlations have also been observed in the $2 \mathrm{~d}$ case [61]. 


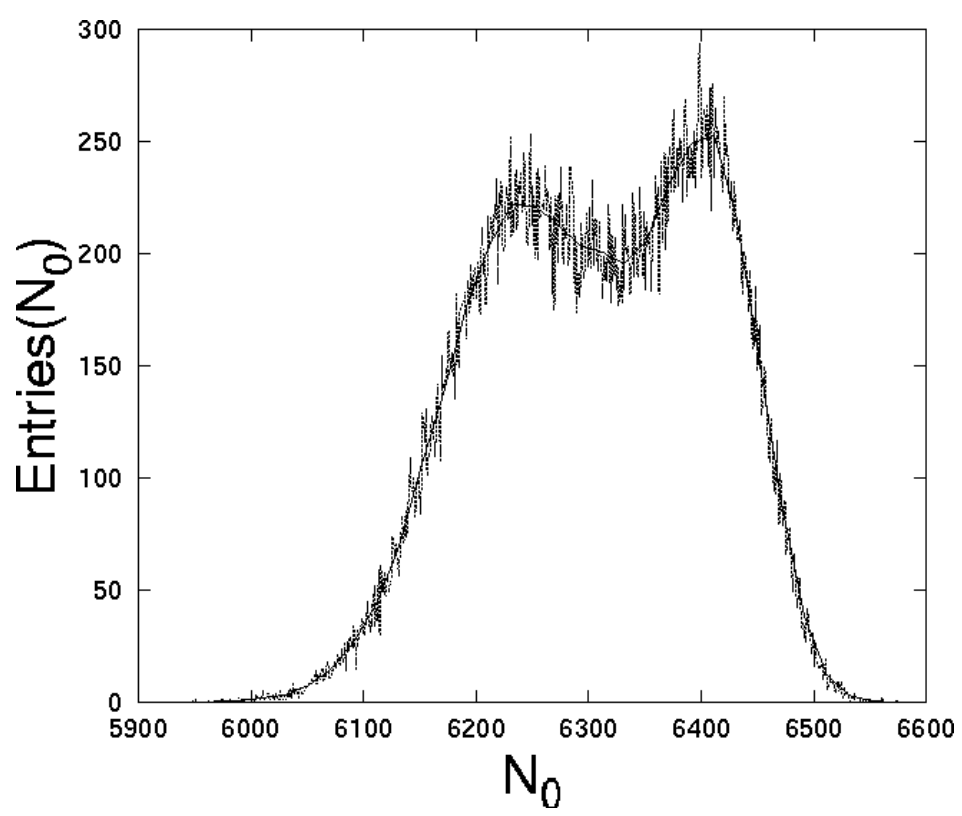

Figure 16. The double peak structure at the phase transion point as a function of the the number of vertices, $N_{0}$, for a fixed number of four-simplices, $N_{4}=32000$.

There is by now overwhelming numerical evidence that the weak- and strong coupling phases are separated by a weak first-order transition [79]. The determination of the order turned out to be difficult, since no hysteresis was observed for finite systems. The best way of identifying the nature of the phase transition is by measuring the distribution of $N_{0}$ in a sample of configurations exactly at the pseudo-critical point. The distribution has two peaks, whose separation grows with the volume. This is shown in Fig. 16. The effect becomes only apparent for sufficiently large systems (of 32000 and 64000 simplices); for smaller volumes the separation cannot be seen.

From the point of view of quantum gravity, this is of course a disappointing result, because there is no new continuum physics associated with the phase transition. The weakness of the transition could be an indication that a continuous phase transition can be reached by enlarging the space of coupling constants, that is, by extending the action $S_{E H}$. Obvious candidates would be terms containing higher powers of the curvature, which have previously been used in quantum Regge calculus [80].

In both $3 \mathrm{~d}$ and $4 \mathrm{~d}$ simplicial gravity, the phases observed on either side of the transition are pathological, in that they do not seem to correspond to an effective quantum geometry of a sensible dimensionality. As already mentioned we find either branched polymers (locally one-dimensional objects) or a condensation of the volume around selected points (yielding an 
object of arbitrarily large dimension). It is surprising that the rich geometric structure observed in two dimensions has all but disappeared. There are potential cures for this, although there is no guarantee that they will lead to the desired quantum theory.

Within the framework of dynamical triangulations, there are basically two ways of "changing the measure", in order to improve this state of affairs. One is by using a modified action, but leaving the sum over geometries untouched. Gravity with a squared scalar-curvature term was investigated in [81], but the numerical results indicated that the phase structure remained unchanged. Note that also in $d=2$, the addition of such terms does not change the universality class of pure gravity, as has been shown both numerically (see, for example, [82]) and analytically [83]. The other possibility is to change the sum to a different class of triangulations. A physically motivated example of this kind is the imposition of a causality constraint on Lorentzian geometries. It does lead to a change of universality class in $d=2$, as we have already seen. There are indications that also in $d>2$ a causality condition acts as an effective regulator for the quantum geometry [84]. Numerical investigations of Lorentzian models in higher dimensions are under way.

Another logical possibility is that only the matter-coupled theory possesses a well-defined scaling limit. Recall that in two dimensions, there is a rather narrow window of physically interesting Euclidean continuum theories, namely, Liouville gravity interacting with conformal fields of charge $0 \leq c \leq 1$. We simply may have been lucky that pure gravity lies within this range.

Matter interactions could in principle suppress the formation of baby universes in higher dimensions, and the inclusion of gauge (vector) fields was suggested as a promising scenario for testing this conjecture [85]. The simplest model of this kind is the non-compact $4 \mathrm{~d}$ Abelian gauge model formulated in [75]. Like on a regular lattice, the gauge fields $A_{i j} \equiv-A_{j i}$ are associated with oriented lattice links $\{i, j\}$. A discretized version $P$ of the field strength tensor $F_{\mu \nu}$ can be expressed in terms of "plaquettes" around (oriented) triangles $\{i j k\}$,

$$
P_{\{i j k\}}=A_{i j}+A_{j k}+A_{k i} .
$$

The action was taken to be

$$
S_{\text {gauge }}=\sum_{\{i j k\}} o(\{i j k\}) P_{\{i j k\}}^{2},
$$

with $o(\{i j k\})$ denoting the order of the triangle $\{i j k\}$. This factor was included to guarantee that whenever $P^{2}$ is constant for all triangles, $S_{\text {gauge }}$ 
is proportional to the volume $N_{4}$ of the manifold. The action has a simple gauge symmetry and is relatively easy to use in numerical simulations, since it is Gaussian in the vector fields. The number $n_{g}$ of gauge fields can be varied, and for $n_{g}=3$ a new "crinkled" phase with $\gamma_{s t r}<0$ was observed, replacing the branched-polymer phase. Moreover, the phase transition seemed to be continuous rather than first-order.

However, it was soon realized that this new phase can be obtained in a model without gauge fields, by adding a geometric term

$$
S_{\Delta}=\frac{n_{g}}{2} \sum_{\{i j k\}} \log o(\{i j k\})
$$

to the pure-gravity action [76]. Since $S_{\Delta}$ is an ultra-local measure term, one would not really expect it to lead to new continuum physics.

The origin of this term can be traced to a somewhat unfortunate choice of gauge action. At first sight, it seems natural to associate the gauge fields with the links of the triangulation, analogous to what is done on regular, flat lattices. This implies that any charged fields which couple to the gauge fields will be located at the vertices. However, note that scalar fields coupled to gravity in all investigations so far have been placed at the centres of the four-simplexes, to ensure that the number of field degrees of freedom grows proportional to the volume. In the case of fluctuating geometry, this need not be the case if the scalar fields are located at the vertices, since $N_{0}$ may grow much slower than $N_{4}$.

Thus, if our primary aim is to compare the effects of gauge fields on geometry with those of the previously studied scalar fields, the complexvalued charged fields should be associated with the four-simplexes of the triangulation, and the gauge fields with the links of the dual lattice. The pure-gauge action is then a sum over the dual plaquettes, which are the natural duals of the triangles in the original triangulation (see [77] for details).

The two models with the gauge potentials $A$ on the links and on the dual links are dual to each other. The theory defined by (137) (on the triangulation) is equivalent to the gauge theory on the dual lattice, if we add by hand a term (138) to the natural gauge action on the dual lattice [77]. Monte Carlo simulations with gauge fields on the dual links without the term (138), as advocated above, have not detected any effect of the gauge fields on the geometry, in line with previous results for scalar fields and Ising spins coupled to gravity.

Putting a more positive spin on this result, the inclusion of the term (138) is the only example of a modification of the action which has led to a genuine change of the geometry. Although it has no obvious physical interpretation and although the crinkled phase is probably not related to 
interesting continuum physics, it does give us some hope of finding a physically well-motivated term which does change the geometry in an interesting way, allowing us to define a continuum theory of gravity.

\section{Outlook}

In these notes we have described in some detail a non-perturbative pathintegral approach to quantum gravity, with and without matter. This "dynamical triangulations" method uses an intermediate regularization, in which the space of all space-time geometries is approximated by a set of simplicial complexes with certain edge length assignments, in the spirit of Regge calculus. For a finite volume (that is, for a complex with a finite number of simplices) and fixed space-time topology, this method yields well-defined, convergent partition functions and propagators. The aim in this approach is to understand the critical behaviour of these statistical systems of fluctuating geometry, and investigate their physical properties in the continuum limit, if it exists.

We believe that this ansatz has a number of virtues that make further research worthwhile. Firstly, it works perfectly in two dimensions, leading to a host of analytical and numerical results. There, it agrees with other discrete or continuum formulations, whenever they can be compared, and in some cases turns out to be even more powerful. This gives us considerable confidence in the validity of the method. Moreover, the two-dimensional construction has served as a useful laboratory for defining diffeomorphisminvariant versions of two-point functions and other field-theoretic observables. Dynamical triangulations as a method can be applied in any dimension $d$, but obviously will lead to very different theories, depending on $d$. Only in $d=4$ do we expect to find local, interacting quantum-gravitational degrees of freedom.

Secondly, as has been shown recently, there is also a Lorentzian version of the formulation, where additional causality restrictions are imposed on the space-time geometries, and a well-defined Wick rotation exists. The absence of a natural notion of "analytic continuation" from Euclidean to Lorentzian signature has for a long time stalled progress in discretized pathintegral approaches to gravity. Surprisingly, in two space-time dimensions the Euclidean and Lorentzian continuum quantum gravity theories are very different. As a consequence of the causality constraint, the "baby-universe" structure of the Euclidean theory is absent in the Lorentzian sector. This leads to a "smoother" quantum geometry, and well-behaved matter coupling even beyond the $c=1$ barrier. Such a behaviour is very desirable in dimension $d>2$, where so far the dominance of "extreme" geometric phases in Euclidean dynamically triangulated models seems to have prevented the 
existence of an interesting continuum limit.

In higher dimensions, much work remains to be done to establish whether our method leads to a well-defined continuum quantum theory. In the Euclidean sector, a suitable change of the discretized measure or the inclusion of different types of matter may still lead to long-range correlations. In the Lorentzian sector, there is a genuine chance that the causality restrictions will lead to a qualitatively different phase structure, including a second-order phase transition. The investigation of the Lorentzian models for $d>2$ has only just begun. However, we do not have to start from scratch when trying to solve the theory, but can use the considerable expertise gathered in the Euclidean simulations. Obviously, quantum gravity will not be solved by numerical methods alone, but we have learned from the example of dynamical triangulations in $2 \mathrm{~d}$ that a mathematically exact analysis and numerical simulations can work hand in hand.

In addition, one can hope for analytical progress in solving the discretized models (which in the case of quantum Regge calculus has always been hampered by the presence of inequalities on the edge lengths, even in $d=2$ ). In terms of dynamical triangulations, quantum gravity has been turned into a well-defined combinatorial problem, which has already been solved exactly in two dimensions. It is also likely that - analogous to the Euclidean case - mean-field methods can help to elucidate the Lorentzian phase structure in higher dimensions. This may have interesting ramifications for the general study of spaces of pseudo-Riemannian geometries.

In summary, the dynamical-triangulations approach is a promising tool for both analytical and numerical investigations of quantum gravity. It allows for a natural inclusion of matter, and is explicitly reparametrizationinvariant. The path integral can be defined for both Euclidean and Lorentzian geometries. In the Lorentzian case, it involves summing over a class of Wick-rotatable space-times with a natural "time"-slicing, reminiscent of the geometric structures appearing in Hamiltonian quantization schemes. We hope that our construction of a Lorentzian path integral will serve as a bridge between the canonical and covariant formulations, benefitting both and improving our understanding of the nature of quantum gravity.

\section{Acknowledgements}

J.A. acknowledges the support of MaPhySto - Center for Mathematical Physics and Stochastics - financed by the National Danish Research Foundation. J.J. was partially supported by the Polish Government KBN grant no. 2p03B01917.

\section{References}


1. T. Banks, W. Fischler, S.H. Shenker and L. Susskind: M theory as a matrix model: a conjecture, Phys. Rev. D 55 (1997) 5112-5128, e-Print Archive: hep-th/9610043.

2. N. Ishibashi, H. Kawai, Y. Kitazawa and A. Tsuchiya: A large-N reduced model as superstring, Nucl. Phys. B 498 (1997) 467-491, e-Print Archive: hep-th/9612115.

3. F. David: Planar diagrams, two-dimensional lattice gravity and surface models, Nucl. Phys. B 257 (1985) 45-58; A model of random surfaces with nontrivial critical behaviour, Nucl. Phys. B 257 (1985) 543-576.

4. J. Ambjørn, B. Durhuus and J. Fröhlich: Diseases of triangulated random surface models, and possible cures, Nucl. Phys. B 257 (1985) 433-449;

J. Ambjørn, B. Durhuus, J. Fröhlich and P. Orland: The appearance of critical dimensions in regulated string theories, Nucl. Phys. B 270 (1986) 457-482.

5. V.A. Kazakov, I.K. Kostov and A.A. Migdal: Critical properties of randomly triangulated planar random surfaces, Phys. Lett. B 157 (1985) 295-300.

6. J. Ambjørn and J. Jurkiewicz: Four-dimensional simplicial quantum gravity, Phys. Lett. B 278 (1992) 42-50.

7. M.E. Agishtein and A.A. Migdal: Simulations of four-dimensional simplicial quantum gravity as dynamical triangulation, Mod. Phys. Lett. A 7 (1992) 1039-1061.

8. J. Ambjørn, B. Durhuus and T. Jonsson, Quantum geometry, Cambridge Monographs on Mathematical Physics, Cambridge University Press, Cambridge, UK, 1997.

9. A. Krzywicki: Random manifolds and quantym gravity, preprint Orsay LPTORSAY-99-51, e-Print Archive: hep-lat/9907012.

10. R. Loll: Discrete approaches to quantum gravity in four dimensions, Living Reviews in Relativity 13 (1998), http://www.livingreviews.org, e-Print Archive: grqc/9805049.

11. J. Ambjørn and R. Loll: Non-perturbative Lorentzian quantum gravity, causality and topology change, Nucl. Phys. B 536 (1998) 407-434, e-Print Archive: hepth/9805108.

12. J. Ambjørn, K.N. Anagnostopoulos and R. Loll: A new perspective on matter coupling in 2d quantum gravity, Phys. Rev. D 60 (1999) 104035, e-Print Archive: hepth/9904012.

13. J. Ambjørn, K.N. Anagnostopoulos and R. Loll: Crossing the $c=1$ barrier in $2 \mathrm{~d}$ Lorentzian gravity, to appear in Phys. Rev. D, e-Print Archive: hep-lat/9909129.

14. C. Teitelboim: Causality versus gauge invariance in quantum gravity and supergravity, Phys. Rev. Lett. 50 (1983) 705-708.

15. L. Bombelli, J. Lee, D. Meyer and R. Sorkin: Space-time as a causal set, Phys. Rev. Lett. 59 (1987) 521-524;

D.P. Rideout and R.D. Sorkin: Classical sequential growth dvnamics for causal sets, Phys. Rev. D 61 (2000) 024002, e-Print Archive: gr-qc/9904062.

16. F. Markopoulou and L. Smolin: Causal evolution of spin networks, Nucl. Phys. B 508 (1997) 409-430, e-Print Archive: gr-qc/9702025; Quantum geometry with intrinsic local causality, Phys. Rev. D 58 (1998) 084032, e-Print Archive: gr-qc/9712067.

17. J. Ambjørn, J. Correia, C. Kristjansen and R. Loll: On the relation between Euclidean and Lorentzian 2d quantum gravity, to appear in Phys. Lett. B, e-Print Archive: hep-th/9912267.

18. H. Kawal, N. Kawamoto, T. Mogami and Y. Watabiki: Transfer matrix formalism for two-dimensional quantum gravity and fractal structures of space-time, Phys. Lett. B 306 (1993) 19-26, e-Print Archive: hep-th/9302133.

19. J. Ambjørn and Y. Watabiki: Scaling in quantum gravity, Nucl. Phys. B 445 (1995) 129-144, e-Print Archive: hep-th/9501049.

20. J. Ambjørn, B. Durhuus and T. Jonsson: A solvable 2-d gravity model with gamma $>$ 0, Mod. Phys. Lett. A 9 (1994) 1221-1228, e-Print Archive: hep-th/9401137.

21. J. Ambjørn, K.N. Anagnostopoulos and R. Loll: On the phase diagram of $2 \mathrm{~d}$ Lorentzian quantum gravity, preprint AEI-1999-20, NBI-HE-99-27 e-Print Archive: hep-lat/9908054. 
22. J. Ambjørn, K.N. Anagnostopoulos and R. Loll: Making the gravitational path integral more Lorentzian, or Life bevond Liouville gravity, preprint AEI-1999-32, NBI-HE-99-43, e-Print Archive: hep-th/9910232.

23. http://www.nbi.dk/ ambjorn/lqg2.

24. M. Carfora and A. Marzuoli: Entropy estimates for simplicial quantum gravity, $J$. Geom. Phys. 16 (1995) 99-119; Holonomy and entropy estimates for dynamically triangulated manifolds, J. Math. Phys. 36 (1995) 6353-6376.

25. J. Ambjørn, M. Carfora and A. Marzuoli: The geometry of dynamical triangulations, Lecture Notes in Physics, New Series, m50, Springer, Berlin, 1997, e-Print Archive: hep-th/9612069.

26. D. Gabrielli: Polymeric phase of simplicial quantum gravity, Phys. Lett. B 421 (1998) 79-85 e-Print Archive: hep-lat/9710055.

J. Ambjørn, M. Carfora, D. Gabrielli and A. Marzuoli: Crumpled triangulations and critical point in 4D simplicial quantum gravity, Nucl. Phys. B 542 (1999) 349-394, e-Print Archive: hep-lat/9806035.

27. P. Bialas, L. Bogacz, Z. Burda and D. Johnston: Finite size scaling of the balls in boxes model, preprint BI-TP-99-41, e-Print Archive: hep-lat/9910047.

P. Bialas, Z. Burda and D. Johnston: Phase diagram of the mean field model of simplicial gravity, Nucl. Phys. B 542 (1999) 413-424, e-Print Archive: gr-qc/9808011; Condensation in the backgammon model, Nucl. Phys. B 493 (1997) 505-516.

S. Catterall, R. Renken and J. Kogut: Singular structure in 4-d simplicial gravity, Phys. Lett. B 416 (1998) 274-280, e-Print Archive: hep-lat/9709007.

28. P. Di Francesco, E. Guitter and C. Kristjansen: Integrable 2d Lorentzian gravity and random walks, to appear in Nucl. Phys. B, e-Print Archive: hep-th/9907084.

29. R.M. Wald: General relativity, University of Chicago Press, Chicago, 1984.

30. R. Sorkin: Time-evolution in Regge calculus, Phys. Rev. D 12 (1975) 385-396; Err. ibid. 23 (1981) 565.

31. R. Nakayama: 2-d quantum gravity in the proper time gauge, Phys. Lett. B 325 (1994) 347-353, e-Print Archive: hep-th/9312158.

32. A. Carlini and J. Greensite: Square root actions, metric signature, and the path integral of quantum gravity, Phys. Rev. D 52 (1995) 6947-6964, e-Print Archive: gr-qc/9502023.

33. A. Carlini and J. Greensite: Fundamental constants and the problem of time, Phys. Rev. D 52 (1995) 936-960, e-Print Archive: gr-qc/9406044.

34. J. Louko and R.D. Sorkin: Complex actions in two-dimensional topology change, Class. Quant. Grav. 14 (1997) 179-203, e-Print Archive: gr-qc/9511023.

35. N. Ishibashi and H. Kawai: String field theory of noncritical strings, Phys. Lett. B 314 (1993) 190-196, e-Print Archive: hep-th/9307045.

36. Y. Watabiki: Construction of noncritical string field theory by transfer matrix formalism in dynamical triangulation, Nucl. Phys. B 441 (1995) 119-166, e-Print Archive: hep-th/9401096.

37. J. Ambjørn, J. Jurkiewicz and Y. Watabiki: Dynamical triangulations, a gateway to quantum gravity?, J. Math. Phys. 36 (1995) 6299-6339, e-Print Archive: hepth/9503108.

38. T. Regge: General relativity without coordinates, Nuovo Cim. A 19 (1961) 558-571.

39. E.A. Bender and E.R. Canfield: The asymptotic number of rooted maps on a surface, J. of Combinatorial Theory A 43 (1986) 244.

40. H.-C. Ren: Matter fields in lattice gravity, Nucl. Phys. B 301 (1988) 661-684.

41. Z. Burda. J. Jurkiewicz and A. Krzywicki, Phys. Rev. D 60 (1999) 105029, e-Print Archive: hep-lat/9905015.

42. M.A. Bershadsky, I.D. Vaisburd and A.A. Migdal: Equivalence of the ising model and the fermion model on a riemann surface, JETP Lett. 43 (1986) 193-196.

43. G. Thorleifsson: Lattice gravity and random surfaces, Nucl. Phys. Proc. Suppl. 73 (1999) 133-145; e-Print Archive: hep-lat/9809131.

M. Bowick: Random surfaces and lattice gravity, Nucl. Phys. Proc. Suppl. 63 (1998) 
77-88; e-Print Archive: hep-lat/9710005

D.A. Johnston: Gravity and random surfaces on the lattice: a review, Nucl. Phys.

Proc. Suppl. 53 (1997) 43-55, e-Print Archive: hep-lat/9607021.

44. S. Bilke, Z. Burda and J. Jurkiewicz: Simplicial quantum gravity on a computer, Comput. Phys. Commun. 85 (1995) 278-292; e-Print Archive: hep-lat/9403017.

S. Catterall: Simulations of dynamically triangulated gravity: an algorithm for arbitrary dimension, Comput. Phys. Commun. 87 (1995) 409-415, e-Print Archive: hep-lat/9405026.

45. U. Wolff: Critical slowing down, Nucl. Phys. B (Proc. Suppl.) 17 (1990) 93-102.

46. J.W. Alexander: The combinatorial theory of complexes, Ann. Mat. 31 (1931) 292.

47. J. Ambjørn, B. Durhuus and T. Jónsson: Summing over all genera for $\mathrm{d}>1$ : a toy model, Phys. Lett. B 244 (1990) 403-412;

P. Bialas and Z. Burda: Phase transition in fluctuating branched geometry, Phys. Lett. B 384 (1996) 75-80, e-Print Archive: hep-lat/9605020;

J.D. Correia, B. Mirza and J.F. Wheater: Branched polymers, complex spins and the freezing transition, Phys. Lett. B 415 (1997) 15-23, e-Print Archive: heplat/9708027

48. J. Ambjørn, S. Jain and G. Thorleifsson: Baby universes in 2-d quantum gravity, Phys. Lett. B 307 (1993) 34-39, e-Print Archive: hep-th/9303149.

49. J. Ambjørn, S. Jain, J. Jurkiewicz and C.F. Kristjansen: Observing 4d baby universes in quantum gravity, Phys. Lett. B 305 (1993) 208-213, e-Print Archive: hepth/9303041.

50. S. Catterall, G. Thorleifsson, M. Bowick and V. John: Scaling and the fractal geometry of two-dimensional quantum gravity, Phys. Lett. B 354 (1995) 58-68, e-Print Archive: hep-lat/9504009.

51. J. Ambjørn, J. Jurkiewicz and Y. Watabiki: On the fractal structure of twodimensional quantum gravity, Nucl. Phys. B 454 (1995) 313-342, e-Print Archive: hep-lat/9507014.

52. B.V. de Bakker and J. Smit: Curvature and scaling in 4-d dynamical triangulation, Nucl. Phys. B 439 (1995) 239-258, e-Print Archive: hep-lat/9407014.

53. J. Ambjørn and J. Jurkiewicz: Scaling in four dimensional quantum gravity, Nucl. Phys. B 451 (1995) 643-676, e-Print Archive: hep-th/9503006.

54. J. Ambjørn, K. Anagnostopoulos, T. Ichihara, L. Jensen, N. Kawamoto, Y. Watabiki and K. Yotsuji: The quantum space-time of $\mathrm{c}=-2$ gravity, Nucl. Phys. B 511 (1998) 673-710. e-Print Archive: hep-lat/9706009: Quantum geometry of topological gravity, Phys. Lett. B 397 (1997) 177-184, hep-lat/9611032.

55. Y. Watabiki: Analytic study of fractal structure of quantized surface in twodimensional quantum gravity, Prog. Theor. Phys. Suppl. 114 (1993) 1-17.

56. J. Ambjørn, D. Boulatov, J.L. Nielsen, J. Rolf and Y. Watabiki: The spectral dimension of 2-d quantum gravity, JHEP 9802:010 (1998), e-Print Archive: hepth/9801099.

57. J. Ambjørn, R. Loll, J.L. Nielsen and J. Rolf: Euclidean and Lorentzian quantum gravity: lessons from two-dimensions, Chaos Solitons Fractals 10 (1999) 177-195, e-Print Archive: hep-th/9806241.

58. T. Jonsson and J.F. Wheater: The spectral dimension of the branched polymer phase of two-dimensional quantum gravity, Nucl. Phys. B 515 (1998) 549-574, ePrint Archive: hep-lat/9710024;

J.D. Correia and J.F. Wheater: The spectral dimension of nongeneric branched polymer ensembles, Phys. Lett. B 422 (1998) 76-81, e-Print Archive: hep-th/9712058.

59. J. Ambjørn and K.N. Anagnostopoulos: Quantum geometry of 2-d gravity coupled to unitary matter, Nucl. Phys. B 497 (1997) 445-478, e-Print Archive: heplat/9701006.

60. J. Ambjørn, K.N. Anagnostopoulos, U. Magnea and G. Thorleifsson: Geometrical interpretation of the KPZ exponents, Phys. Lett. B 388 (1996) 713-719, e-Print Archive: hep-lat/9606012. 
61. J. Ambjørn, P. Bialas and J. Jurkiewicz: Connected correlators in quantum gravity, JHEP 9902:005 (1999), e-Print Archive: hep-lat/9812015.

62. N. Ishibashi and H. Kawai: String field theory of $\mathrm{c}<1$ noncritical strings, Phys. Lett. B 322 (1994) 67-78, e-Print Archive: hep-th/9312047.

63. J. Ambjørn, K.N. Anagnostopoulos, J. Jurkiewicz and C.F. Kristjansen: The concept of time in 2-d gravity, JHEP 9804:016 (1998), e-Print Archive: hep-th/9802020.

64. U. Pachner: Bistellare Äquivalenz kombinatorischer Mannigfaltigkeiten, Arch. Math. 30 (1978) 89; Konstruktionsmethoden und das kombinatorische Homöomorphieproblem für Triangulationen kompakter semilinearer Mannigfaltigkeiten, $A b h$. Math. Sem. Univ. Hamburg 57 (1986) 69-85; P.L. homeomorphic manifolds are equivalent by elementary shellings, Europ. J. Combinatorics 12 (1991) 129-145.

65. M. Gross and S. Varsted: Elementary moves and ergodicity in d-dimensional simplicial quantum gravity, Nucl. Phys. B 378 (1992) 367-380.

66. A. Nabutovsky and R. Ben-Av: Noncomputability arising in dynamical triangulation model of four-dimensional quantum gravity, Commun. Math. Phys. 157 (1993) 93-98.

67. A. Thompson: Thin position and the recognition problem for $\mathrm{S}^{3}$, Math. Res. Lett. 1 (1994) 613.

68. F. Acquistapace, R. Benedetti and F. Broglia: Effectiveness and non-effectiveness in semialgebraic and PL geometry, Invent. Math. 102 (1990) 141.

69. J. Ambjørn and J. Jurkiewicz: Computational ergodicity of $\mathrm{S}^{4}$, Phys. Lett. B 345 435-440, e-Print Archive: hep-lat/9411008.

70. B.V. de Bakker: Absence of barriers in dynamical triangulation, Phys. Lett. B 348 35-38, e-Print Archive: hep-lat/9411070.

71. D.V. Boulatov and A. Krzywicki: On the phase diagram of three-dimensional simplicial quantum gravity, Mod. Phys. Lett. A 6 (1991) 3005-3014.

J. Ambjørn and S. Varsted: Three-dimensional simplicial quantum gravity, Nucl. Phys. B 373 (1992) 557-580.

J. Ambjørn, D.V. Boulatov, A. Krzywicki and S. Varsted: The vacuum in threedimensional simplicial quantum gravity, Phys. Lett. B 276 (1992) 432-436;

M.E. Agishtein and A.A. Migdal: Three-dimensional quantum gravity as dynamical triangulation, Mod. Phys. Lett. A 6 (1991) 1863-1884;

R.L. Renken, S.M. Catterall and J.B. Kogut: Phase structure of dynamical triangulation models in three-dimensions, Nucl. Phys. B 523 (1998) 553-568, e-Print Archive: hep-lat/9712011.

72. J. Ambjørn, Z. Burda, J. Jurkiewicz and C.F. Kristjansen: 3-d quantum gravity coupled to matter, Phys. Lett. B 297 (1992) 253-260, e-Print Archive: hep-lat/9205021: R.L. Renken, S.M. Catterall and J.B. Kogut: Three-dimensional quantum gravity coupled to ising matter, Nucl. Phys. B 389 (1993) 601-610, e-Print Archive: heplat/9205029

73. J. Ambjørn and J. Jurkiewicz: Four-dimensional simplicial quantum gravity, Phys. Lett. B 278 (1992) 42-50; Scaling in four dimensional quantum gravity, Nucl. Phys. B 451 (1995) 643-676, e-Print Archive: hep-th/9503006;

M.E. Agishtein and A.A. Migdal: Critical behavior of dynamically triangulated quantum gravity in four-dimensions, Nucl. Phys. B 385 (1992) 395-412; e-Print Archive: hep-lat/9204004;

S. Catterall, J. Kogut and R. Renken: Phase structure of four-dimensional simplicial quantum gravity. Phys. Lett. B 328 (1994) 277-283; e-Print Archive: heplat/9401026.

74. S. Catterall, R. Renken and J. Kogut: Singular structure in 4-d simplicial gravity, Phys. Lett. B 416 (1998) 274-280, e-Print Archive: hep-lat/9709007;

S. Catterall, G. Thorleifsson, J. Kogut and R. Renken: Singular vertices and the triangulation space of the d sphere, Nucl. Phys. B 468 (1996) 263-276, e-Print Archive: hep-lat/9512012; 
T. Hotta, T. Izubuchi and J. Nishimura: Singular vertices in the strong coupling phase of four-dimensional simplicial gravity, Prog. Theor. Phys. 94 (1995) 263-270, e-Print Archive: hep-lat/9709073.

P. Bialas, Z. Burda, B. Petersson and J. Tabaczek: Appearance of mother universe and singular vertices in random geometries, Nucl. Phys. B 495 (1997) 463-476, e-Print Archive: hep-lat/9608030.

75. S. Bilke, Z. Burda, A. Krzywicki, B. Petersson, J. Tabaczek and G. Thorleifsson: 4-d simplicial quantum gravity interacting with gauge matter fields, Phys. Lett. B 418 (1998) 266-272, e-Print Archive: hep-lat/9710077.

76. S. Bilke, Z. Burda, A. Krzywicki, B. Petersson, J. Tabaczek and G. Thorleifsson: 4-d simplicial quantum gravity: matter fields and the corresponding effective action, Phys. Lett. B 432 (1998) 279-286, e-Print Archive: hep-lat/9804011.

77. J. Ambjørn, K.N. Anagnostopoulos and J. Jurkiewicz: Abelian gauge fields coupled to simplicial quantum gravity, JHEP 9908:016 (1999), e-Print Archive: heplat/9907027

78. B.V. de Bakker and J. Smit: Two point functions in 4-d dynamical triangulation, Nucl. Phys. B 454 (1995) 343-356, e-Print Archive: hep-lat/9503004.

79. P. Bialas, Z. Burda, A. Krzywicki and B. Petersson: Focusing on the fixed point of 4-d simplicial gravity, Nucl. Phys. B 472 (1996) 293-308, e-Print Archive: heplat/9601024.

B.V. de Bakker: Further evidence that the transition of 4-d dvnamical triangulation is first order, Phys. Lett. B 389 238-242, e-Print Archive: hep-lat/9603024.

80. H.W. Hamber: Phases of simplicial quantum gravity in four-dimensions: estimates for the critical exponents, Nucl. Phys. B 400 (1993) 347-389; On the gravitational scaling dimensions, preprint Irvine UCI-99-20, e-Print Archive: hep-th/9912246.

81. J. Ambjørn, J. Jurkiewicz and C.F. Kristjansen: Quantum gravity, dynamical triangulation and higher derivative regularization, Nucl. Phys. B 393 (1993) 601-632, e-Print Archive: hep-th/9208032.

82. J. Jurkiewicz and Z. Tabor: Critical properties of the two-dimensional gravity with the $\mathrm{R}^{2}$ action. Acta Phys. Polon. B 25 (1994) 1087-1097.

83. V.A. Kazakov, M. Staudacher and T. Wynter: Exact solution of discrete twodimensional $\mathrm{R}^{2}$ gravity, Nucl. Phys. B 471 (1996) 309-333, e-Print Archive: hep$\operatorname{th} / 9601069$.

84. J. Ambjørn, J. Jurkiewicz and R. Loll, to be published.

85. I. Antoniadis, P.O. Mazur and E. Mottola: Scaling behavior of quantum fourgeometries, Phys. Lett. B 323 (1994) 284-291, e-Print Archive: hep-th/9301002; Conformal symmetry and central charges in four-dimensions, Nucl. Phys. B 388 (1992) 627-647, e-Print Archive: hep-th/9205015;

I. Antoniadis and E. Mottola: 4-d quantum gravity in the conformal sector, Phys. Rev. D 45 (1992) 2013-2025. 